\title{
THE FINE MODULI SPACE OF REPRESENTATIONS OF CLIFFORD ALGEBRAS
}

\author{
EMRE COSKUN
}

\begin{abstract}
Given a fixed binary form $f(u, v)$ of degree $d$ over a field $k$, the associated Clifford algebra is the $k$-algebra $C_{f}=k\{u, v\} / I$, where $I$ is the two-sided ideal generated by elements of the form $(\alpha u+\beta v)^{d}-f(\alpha, \beta)$ with $\alpha$ and $\beta$ arbitrary elements in $k$. All representations of $C_{f}$ have dimensions that are multiples of $d$, and occur in families. In this article we construct fine moduli spaces $U=U_{f, r}$ for the irreducible $r d$-dimensional representations of $C_{f}$ for each $r \geq 2$. Our construction starts with the projective curve $C \subset \mathbb{P}_{k}^{2}$ defined by the equation $w^{d}=f(u, v)$, and produces $U_{f, r}$ as a quasiprojective variety in the moduli space $\mathcal{M}\left(r, d_{r}\right)$ of stable vector bundles over $C$ with rank $r$ and degree $d_{r}=r(d+g-1)$, where $g$ denotes the genus of $C$.
\end{abstract}

\section{INTRODUCTION}

Let $f$ be a binary form of degree $d$ over a field $k$. The Clifford algebra $C_{f}$ associated to $f$ is the quotient of the tensor algebra on two variables by the twosided ideal generated by $\left\{(\alpha u+\beta v)^{d}-f(\alpha, \beta) \mid \alpha, \beta \in k\right\}$. We will be interested in the case of degree $d>3$. We also assume that $f(u, v)$ is nondegenerate, that is, $f$ has no repeated roots over the algebraic closure of $k$.

The structure and representations of Clifford algebras have been a subject of study in many recent papers. The degree 2 case is classical; for an overview of the subject, see [11] and [16. The degree 3 case was examined by Haile in [6]. Assuming that the characteristic of the base field is not 2 or 3 , and that $f(u, v)$ is nondegenerate, he proved that $C_{f}$ is an Azumaya algebra (see Section 2.3) over its center. He also proved that the center is isomorphic to the coordinate ring of an affine elliptic curve $J$. The curve $J$ is the Jacobian of $w^{3}=f(u, v)$ and the affine elliptic curve is the complement of the identity point in $J$.

Next we describe what is known for $d>3$. Let $C$ be the curve over $k$ defined by the equation $w^{d}=f(u, v)$ in $\mathbb{P}_{k}^{2}$, and let $g$ denote its genus. Since $f$ is assumed to be nondegenerate, the curve $C$ is nonsingular, and $g=(d-1)(d-2) / 2$. Haile and Tesser proved in 21] that the dimensions of representations of $C_{f}$ are divisible by $d$. Van den Bergh proved in 22. (assuming that the base field $k$ is algebraically closed of characteristic 0) that the equivalence classes of $r d$-dimensional representations of the Clifford algebra $C_{f}$ are in one-to-one correspondence with vector bundles $E$ over $C$ having rank $r$, degree $r(d+g-1)$ such that $H^{0}(E(-1))=0$. These vector

Date: November 10, 2018.

2000 Mathematics Subject Classification. Primary: 14D22, 14H60, 16G99, 16H05.

Key words and phrases. Clifford algebra, moduli space, representation.

I am indebted to my thesis advisor Rajesh Kulkarni for his help on this project. I also want to thank Ajneet Dhillon for several valuable conversations involving the last section, and the referee for making valuable suggestions. 
bundles are always semistable and the stable bundles correspond to the irreducible representations. Then, Haile and Tesser proved in [21] that $\widetilde{C}_{f}=C_{f} / \cap \eta$, where $\eta$ runs over the kernels of the dimension $d$ representations, is Azumaya over its center. The center of $\widetilde{C}_{f}$ is then the fine moduli space of $d$-dimensional representations (hence $r=1$ ) of $C_{f}$. Kulkarni then proved in [9] that this center is the affine coordinate ring of the complement of a $\Theta$-divisor in the space $P i c_{C / k}^{d+g-1}$ of degree $d+g-1$ line bundles over $C$.

This article generalizes Kulkarni's work to the higher rank case, $r \geq 2$; under the assumption that the characteristic of $k$ does not divide $d$. We begin with Van den Bergh's correspondence between equivalence classes of $r d$-dimensional representations of $C_{f}$ and semistable vector bundles over $C$ of rank $r$ and degree $r(d+g-1)$, which can be described as follows. Consider a representation $\phi: C_{f} \rightarrow M_{r d}(k)$. Set $\alpha_{u}=\phi(u)$ and $\alpha_{v}=\phi(v)$. Then we define a map from $S=k[u, v, w] /\left(w^{d}-f(u, v)\right)$ to $M_{r d}(k[u, v])$ by sending $u$ to $u I_{r d}, v$ to $v I_{r d}$, and $w$ to $u \alpha_{u}+v \alpha_{v}$. This makes $\bigoplus_{r d} k[u, v]$ into a graded $S$-module. It can be proven that the corresponding coherent sheaf is a vector bundle. In this way we get a rank $r$ vector bundle $\mathcal{E}$ over $C$ such that $q_{*} \mathcal{E} \cong \mathcal{O}_{\mathbb{P}_{k}^{1}}^{r d}$, where $q: C \rightarrow \mathbb{P}_{k}^{1}$ is the map defined by the inclusion $k[u, v] \rightarrow S$. The condition $H^{0}(E(-1))=0$ is equivalent to the condition that $q_{*} \mathcal{E} \cong \mathcal{O}_{\mathbb{P}_{k}^{1}}^{r d}$.

The moduli problem we solve in this article can be stated as follows: Define a contravariant functor $\mathcal{R e p}_{r d}\left(C_{f},-\right)$ from the category of $k$-schemes to the category of sets by sending a $k$-scheme $S$ to the set of equivalence classes of $r d$-dimensional irreducible $S$-representations of $C_{f}$. (For the definition of an $S$-representation of $C_{f}$, see Definition 2.13.) Procesi proved that this functor is representable by a scheme $U$ (see Theorem 1.8 in Chapter 4 of [17].) Unfortunately, Procesi's method gives no geometric description of $U$. In this article, we prove that $U$ is isomorphic to an open subset of the coarse moduli space $\mathcal{M}(r, r(d+g-1))$ of stable vector bundles of rank $r$ and degree $r(d+g-1)$ over $C$. Using this geometric description, we construct the universal representation $\mathcal{A}$ of $C_{f}$ over $U$.

More explicitly, the universal representation of $C_{f}$ of a given dimension $r d$ is a $k$-algebra homomorphism $\psi: C_{f} \rightarrow H^{0}(\mathcal{A})$, where $\mathcal{A}$ is a sheaf of Azumaya algebras of rank $(r d)^{2}$ defined over $U$. The base variety $U$ is the open subset of $\mathcal{M}(r, r(d+g-1))$ consisting of stable vector bundles $E$ such that $H^{0}(E(-1))=0$. The sheaf $\mathcal{A}$ is constructed as follows. There is a Quot scheme $Q$ (see Theorem 2.6), that parametrizes the quotients of the trivial vector bundle of large enough rank $N$ over $C$, having rank $r$ and degree $r(d+g-1)$, and there is a universal bundle $\mathcal{E}$ over $C \times Q$. We take the open subset $\Omega$ of $Q$ consisting of stable vector bundles $E$ with $H^{0}(E(-1))=0$. We prove in Lemma 3.4 that the pushforward of $\mathcal{E}$ to $\Omega$ under the projection map $\pi: C \times \Omega \rightarrow \Omega$ is a rank $r d$ vector bundle. The algebraic group $G L(N)$ acts on $\Omega$ and also on $\pi_{*} \mathcal{E}$. The stabilizer of a point in $\Omega$ under this action is the group of scalar matrices, so the action of $G L(N)$ on $\Omega$ descends to an action of $P G L(N)$. But the scalar matrices act as scalar multiplication on $\pi_{*} \mathcal{E}$. So we get a $P G L(N)$-action on $\mathcal{E} n d\left(\pi_{*} \mathcal{E}\right)$. The resulting Geometric Invariant Theory quotient is the variety $U$ together with a sheaf of algebras $\mathcal{A}$ on it. We then construct the homomorphism $\psi: C_{f} \rightarrow H^{0}(\mathcal{A})$.

The main theorem of this article is as follows: 
Theorem 1.1. (Main Theorem) Let $k$ be an algebraically closed base field. $\mathcal{R} e p_{r d}\left(C_{f},-\right)$ is represented by the pair $(\psi, \mathcal{A})$ described above.

For the proof, we construct an isomorphism between $(\psi, \mathcal{A})$ and the universal representation in Procesi's theorem. As mentioned above, Procesi showed that the functor $\operatorname{Rep}_{r d}\left(C_{f},-\right)$ is representable, that is, there is a universal representation $(\Psi, \mathcal{B})$ consisting of a scheme $T$, a sheaf of Azumaya algebras $\mathcal{B}$ over $T$, and a $k$-algebra homomorphism $\Psi: C_{f} \rightarrow H^{0}(\mathcal{B})$. Since we have an irreducible representation $(\psi, \mathcal{A})$ over $U$, we obtain a map $\alpha: U \rightarrow T$ such that $\alpha^{*}(\Psi, \mathcal{B}) \cong(\psi, \mathcal{A})$. The idea of the proof is to construct an inverse to $\alpha$. To do this, we first consider irreducible representations of the type $\left(\phi, \mathcal{E} n d\left(\mathcal{E}^{S}\right)\right)$, where $\mathcal{E}^{S}$ is a vector bundle of rank $r d$ over $S$. We construct an associated vector bundle in Lemma 4.2. By the coarse moduli property of $\mathcal{M}(r, r(d+g-1))$, this gives us a map $f: S \rightarrow U$. We use this to construct a morphism $\beta: T \rightarrow U$. We then prove that $\alpha$ and $\beta$ are inverses, and hence that $(\psi, \mathcal{A})$ represents $\mathcal{R e p}_{r d}\left(C_{f},-\right)$. This finishes the proof of the theorem.

Let $B$ be a $k$-algebra. As mentioned above, the functor $\mathcal{R} e p_{n}(B,-)$ that assigns the set of irreducible $S$-representations of $B$ to the $k$-scheme $S$ is representable by Procesi's theorem. However, explicit descriptions of the scheme Rep $p_{n}$ that represents $\mathcal{R} e p_{n}$ are rare. The current article is of interest in this direction because it provides an explicit description of $\operatorname{Re}_{n}$ for the Clifford algebra as an open subvariety of the quasiprojective variety that is the coarse moduli space of stable, rank $r$ and degree $r(d+g-1)$ vector bundles over the curve $C$.

Further questions can be asked about this universal representation. $\mathcal{A}$ gives a class in the Brauer group $\operatorname{Br}(U)$. Since the Brauer group is torsion, it is natural to ask what the period and index of this class (as defined in Section 2.3) are. It is known that the period always divides the index, and the set of primes dividing both of them is the same. Hence the index divides a power of the period. The period-index problem is the problem of computing this power. This is part of an ongoing project.

Second, it is an interesting question to examine the representations of Clifford algebras of ternary forms. By the results of Van den Bergh in 22, these correspond to vector bundles over a surface $X$ in $\mathbb{P}^{3}$ defined by the equation $z^{d}=f(u, v, w)$, whose direct images under the natural projection map $X \rightarrow \mathbb{P}^{2}$ is a trivial vector bundle. These will be studied in future articles.

Conventions and notation. Let $k$ be a perfect, infinite base field with characteristic 0 or not dividing $d$. A variety means a separated scheme of finite type over $k$. A variety of dimension 1 is called a curve.

- All rings have an identity element.

- All schemes are locally Noetherian over $k$ and all morphisms are locally of finite type over $k$.

- The terms line bundle and invertible sheaf are used interchangeably.

- $\mathcal{M}(r, d)$ denotes the coarse moduli space of stable vector bundles of rank $r$ and degree $d$ over a curve $C$.

- For any vector bundle $\mathcal{E}, \chi(\mathcal{E})$ denotes the Euler characteristic of $\mathcal{E}$.

- The projection of a fiber product onto the $i^{\text {th }}$ component $X_{i}$ is denoted $p_{i}, \pi_{i}, p_{X_{i}}$ or $\pi_{X_{i}}$. When no subscript is indicated, $\pi$ is the canonical map from $C \times Y \rightarrow Y$ for a variety $Y$. 
- For technical reasons, we assume throughout the article that the binary form $f(x, y)$ has no repeated factors over the algebraic closure of the base field $k$ and that the characteristic of $k$ does not divide $d$.

- $q$ denotes the canonical map from $C$ to $\mathbb{P}^{1}$, where $C$ is the curve $w^{d}=f(u, v)$ in $\mathbb{P}^{2}$. $q_{S}$ denotes the map $q \times i d_{S}: C \times S \rightarrow \mathbb{P}^{1} \times S$ for any scheme $S$, and $p_{S}: \mathbb{P}^{1} \times S \rightarrow S$ is the natural projection.

- For any coherent sheaf $\mathcal{F}$ over a scheme $X$, we denote the $i^{t h}$ cohomology of $\mathcal{F}$ by $H^{i}(\mathcal{F})$ whenever $X$ is clear from the context.

- For a closed point $y$ in a scheme $S$, and for a vector bundle $\mathcal{E}$ over $C \times S, \mathcal{E}_{y}$ denotes the pull-back of $\mathcal{E}$ under the canonical map $i d \times i_{y}: C \times \operatorname{Spec} k(y) \rightarrow$ $C \times S$.

The following lemma will be useful in proving the main theorem.

Lemma 1.2. Let $Y$ be a reduced quasi-projective variety over an algebraically closed field $k$. Let $f: Y \rightarrow Y$ be a morphism of $k$-varieties that is the identity on closed points. Then $f$ is the identity morphism.

Proof. First we prove that $f$ is the identity on all points. Let $\xi$ be a non-closed point. Assume that $f(\xi)=\eta \neq \xi$. Then since closed irreducible subsets have unique generic points in a scheme (see 2.1.2 and 2.1.3 Chapitre 0 and Corollaire 1.1.8 Chapitre 1, [5] Vol. 1 for more details), we have $\bar{\xi} \neq \bar{\eta}$, where $\bar{\xi}$ and $\bar{\eta}$ denote the closures of $\xi$ and $\eta$, respectively. There are two cases to consider:

Case 1: $\bar{\xi} \not \Phi \bar{\eta}$. In this case, $\bar{\xi} \backslash \bar{\eta}$ is a nonempty open subset $V$ of $\bar{\xi}$. Since $V$ is a quasi-projective variety over $k$, it has a closed point. Pick a closed point $y \in \bar{\xi} \backslash \bar{\eta}$. Then $f^{-1}(\bar{\eta})$ is a closed set that contains $\xi$, and hence it contains $\bar{\xi}$ and in particular $y$, a contradiction.

Case 2: $\bar{\xi} \subsetneq \bar{\eta}$. Since this is a proper inclusion, the dimension of $\bar{\eta}$ is strictly greater than the dimension of $\bar{\xi}$. But since $k(\eta)$ is a subfield of $k(\xi)$, this is a contradiction for dimension reasons.

Since $f$ is the identity map on points, it maps affine open subsets to affine open subsets.

Now since any reduced quasi-projective variety can be covered by reduced open affine varieties, without loss of generality we may assume that $Y$ affine, and hence it is the spectrum of a finitely generated reduced $k$-algebra $A=A(Y)=k\left[T_{1}, \ldots, T_{n}\right] / I(Y)$. Let $f$ be induced by the morphism of rings $\phi: A \rightarrow A$.

Let $a \in A$. We may view $a$ as a morphism $a: Y \rightarrow \mathbb{A}^{1}$. Then the composition $a \circ f: Y \rightarrow Y \rightarrow \mathbb{A}^{1}$ corresponds to $\phi(a) \in A$. Since $f$ is the identity on points; as morphisms $Y \rightarrow \mathbb{A}^{1}, a$ and $\phi(a)$ take the same value on all the points on $Y$. This means that, for any prime ideal $\mathfrak{p}$ of $A$, we have $a-\phi(a) \in \mathfrak{p}$. Since $A$ is reduced, this means that $a-\phi(a)=0$ and hence $\phi$ is the identity morphism.

\section{Preliminaries}

In this section, we will review the results to be used in this article.

2.1. Moduli of vector bundles over curves. Here we review how to construct the moduli spaces of vector bundles over curves. (For more information, see [15], [10] and [2.) Let $C$ be a nonsingular irreducible projective curve of genus $g \geq 2$ and let $E$ be a vector bundle over $C$. The degree $\operatorname{deg}(E)$ is defined to be the degree of the determinant line bundle $\operatorname{det}(E)$ of $E$, and can be any integer. A family of 
vector bundles over $C$ parametrized by a scheme $S$ is a vector bundle over $C \times S$ that is flat over $S$; and an isomorphism of families is just an isomorphism of vector bundles over $C \times S$. For a family $E$ of vector bundles parametrized by $S$ and $s \in S$, we denote by $E_{s}$ the fiber of $E$ over $s$.

To be able to define the moduli space of vector bundles over $C$ with $\operatorname{rank} r \geq 2$ and degree $D$ as a variety, we have to introduce extra conditions on the vector bundles. To do that, we define the slope of a vector bundle $E$ over $C$ as $\mu(E)=$ $\operatorname{deg}(E) / r k(E)$. Then we have the following:

Definition 2.1. A vector bundle $E$ over $C$ is stable (semistable) if, for every nontrivial subbundle $F$ with $F \neq E$,

$$
\mu(F)<\mu(E) \quad(\leq)
$$

With these definitions in place, we can now state the main results about the moduli space of vector bundles over $C$ :

Theorem 2.2. There exist coarse moduli spaces $\mathcal{M}(r, D)$ and $\mathcal{M}^{s s}(r, D)$ for stable and semistable bundles of rank $r$ and degree $D$ over any nonsingular irreducible projective curve $C$ of genus $g \geq 2 . \mathcal{M}(r, D)$ is a nonsingular quasiprojective variety that is contained in $\mathcal{M}^{s s}(r, D)$, which is a projective variety. $\mathcal{M}^{s s}(r, D)$ is normal, and its singular locus is given by $\mathcal{M}^{s s}(r, D) \backslash \mathcal{M}(r, D)$. The dimension of these moduli spaces is equal to $r^{2}(g-1)+1$. Moreover, $\mathcal{M}(r, D)$ is a fine moduli space if and only if $\operatorname{gcd}(r, D)=1$.

The points of $\mathcal{M}(r, D)$ correspond to stable vector bundles of rank $r$ and degree $D$ over $C$. To describe the points of $\mathcal{M}^{s s}(r, D) \backslash \mathcal{M}(r, D)$, we note that for any semistable bundle $E$ over $C$, there is a sequence of subbundles

$$
E_{1} \subseteq E_{2} \subseteq \ldots E_{n}=E
$$

such that $E_{1}, E_{2} / E_{1}, \ldots, E_{n} / E_{n-1}$ are all stable with slopes equal to $\mu(E)$. Moreover, it can be shown that the bundle $g r E=E_{1} \oplus E_{2} / E_{1} \oplus \ldots \oplus E_{n} / E_{n-1}$ is determined up to isomorphism by $E$. (This is called the Jordan-Hölder filtration.) We have

Proposition 2.3. Two semistable bundles $E$ and $E^{\prime}$ determine the same point of $\mathcal{M}^{s s}(r, D)$ if and only if $g r E \cong g r E^{\prime}$.

Let us now recall how the coarse moduli space $\mathcal{M}(r, D)$ of stable vector bundles of rank $r$ and degree $D$ is constructed. The following lemma is crucial:

Lemma 2.4. Let $E$ be a semistable vector bundle over $C$ of rank $r$ and degree $D$, and suppose that $D>r(2 g-1)$. Then

(1) $H^{1}(E)=0$

(2) $E$ is generated by its sections.

For large enough $m$, the degree of $E(m)=E \otimes \mathcal{O}_{C}(m)$ is greater than $r(2 g-1)$ and the lemma allows us to write it as a quotient of a trivial vector bundle $\mathbb{E}=$ $\bigoplus_{N} \mathcal{O}_{C}$ over $C$. To determine the necessary rank $N$ of this trivial vector bundle, which is the same as $h^{0}(E(m))$, we can simply use the Riemann-Roch theorem:

Theorem 2.5. (Riemann-Roch) Let $E$ be a vector bundle over a curve $C$ with genus $g \geq 2$. Then we have:

$$
\chi(E)=h^{0}(E)-h^{1}(E)=(1-g) r k(E)+\operatorname{deg}(E) .
$$


Let $r=r k(E)$, and $d=\operatorname{deg}\left(\mathcal{O}_{C}(1)\right)$ where $\mathcal{O}_{C}(1)$ is a fixed very ample line bundle on $C$. Note that using the Riemann-Roch theorem, we have

$$
\chi(E(m))=(1-g) r+\operatorname{deg}(E(m))=(1-g) r k(E)+\operatorname{deg}(E)+r d m .
$$

Hence specifying the rank and degree of a vector bundle determines its Hilbert polynomial $P(m)$.

Let $\mathbb{E}=\bigoplus_{N} \mathcal{O}_{C}$ be as above, and let $P$ be a linear polynomial with integer coefficients. We denote by $Q=Q(\mathbb{E}, P)$ the family of all coherent sheaves $\mathcal{F}$ on $C$ together with a surjection $\mathbb{E} \rightarrow \mathcal{F}$ such that the Hilbert polynomial of $\mathcal{F}$ is $P$. Now, the main tool in the construction is the following theorem of Grothendieck. (See Théorème 3.1, [4.) The statement below is from Theorem 6.1, [20].

Theorem 2.6. (Grothendieck) There is a unique projective algebraic variety structure on $Q=Q(\mathbb{E}, P)$, and a surjection $\theta: p_{1}^{*}(\mathbb{E}) \rightarrow \mathcal{E}$ of coherent sheaves on $C \times Q$, where $p_{1}$ is the canonical projection $C \times Q \rightarrow C$, such that:

1: $\mathcal{E}$ is flat over $Q$;

2: the restriction of the homomorphism $\theta: p_{1}^{*}(\mathbb{E}) \rightarrow \mathcal{E}$ to $C \times q \cong C, q \in Q$; when viewed as a surjection $\left.\mathbb{E} \rightarrow \mathcal{E}\right|_{C \times q}$, corresponds to the element of $Q(\mathbb{E}, P)$ represented by $q$;

3: given a surjection $\phi: p_{1}^{*}(\mathbb{E}) \rightarrow G$ of coherent sheaves on $C \times T$, where $T$ is an algebraic scheme such that $G$ is flat over $T$, and the Hilbert polynomial of the restriction of $G$ to $C \times t \cong C$ is $P$, there exists a unique morphism $f: T \rightarrow Q$ such that $\phi: p_{1}^{*}(\mathbb{E}) \rightarrow G$ is the inverse image of $\theta: p_{1}^{*}(\mathbb{E}) \rightarrow \mathcal{E}$ by the morphism $f$.

Note that the group $G L(N)$ may be identified with the group of automorphisms of $\mathbb{E}$, hence $G L(N)$ acts on $Q$, and also on the sheaf $\mathcal{E}$. The action of $G L(N)$ on $Q$ goes down into an action of $P G L(N)$, but it does not go into an action of $P G L(N)$ on $\mathcal{E}$. The scalar multiples of identity act as scalar multiplication on $\mathcal{E}$. (This is the reason why the moduli space for vector bundles over curves is not fine in general.)

Let $R^{s}$ be the subset of $Q$ consisting of those $x$ in $Q$ for which the bundle $\mathcal{E}_{x}$ is stable. This is an open subset of $Q$ on which $P G L(N)$ acts freely and hence has a quotient. This quotient is the moduli space $\mathcal{M}\left(r, D^{\prime}\right)$.

Now we discuss some properties of $\mathcal{E}$. We will assume that the rank $r$ and the degree $D$ are given, and that the vector bundles can be twisted by $\mathcal{O}_{C}(m)$ to make their degrees $D^{\prime}$ larger than $r(2 g-1)$, as required in 2.4. By Theorem 5.3, 15, the bundle $\mathcal{E}$ has the local universal property for families of bundles of rank $r$ and degree $D^{\prime}$ which satisfy conditions (1) and (2) in 2.4 Let $\mathcal{F}$ be a family of vector bundles over $C$ with rank $r$, degree $D^{\prime}$ and satisfying (1) and (2); parametrized by a scheme $T$ and flat over $T$. Then we can cover $T$ with open subsets $T_{i}$ and we can find maps $f_{i}: T_{i} \rightarrow Q$ such that $\mathcal{F}$ is isomorphic to $\left(i d_{C} \times f_{i}\right)^{*} \mathcal{E}$. We do not require the maps $f_{i}$ to be unique.

Let $U$ denote the subset of $\mathcal{M}(r, r(d+g-1))$ consisting of vector bundles $E$ over $C$ such that $H^{0}(E(-1))=0$. We prove that this is a nonempty open subset. The fact that it is nonempty was proven in 22, Theorem 2.4. To prove that it is open, we look at the subset $\Omega$ of $R^{s}$ consisting of vector bundles with the same property. This is a smooth subset. (For details, see [15] and [10.) Then $P G L(N)$ acts freely on $\Omega$ and we can take the GIT-quotient to construct $U$.

Lemma 2.7. $\Omega$ is open in $R^{s}$. 
Proof. Note that the second projection $C \times R^{s} \rightarrow R^{s}$ is a projective morphism. Since any affine subset of $R^{s}$ is noetherian, we can restrict to an affine open subset after choosing an affine open cover. We also note that $\mathcal{E} \otimes p_{C}^{*} \mathcal{O}_{C}(-m-1)$ is a coherent sheaf on $C \times R^{s}$ and is flat over $R^{s}$, by Grothendieck's theorem. That the set $\Omega$ is open in $R^{s}$ now follows from the Semicontinuity Theorem, Thm. 12.8, [8.

2.2. The Clifford algebra and its representations. Let $f(u, v)$ be a binary form of degree $d$ over $k$. We define the Clifford algebra of $f$, denoted $C_{f}$ to be the associative $k$-algebra $k\{u, v\} / I$, where $I$ is the two-sided ideal generated by elements of the form $(\alpha u+\beta v)^{d}-f(\alpha, \beta)$, where $\alpha$ and $\beta$ are arbitrary elements of $k$. A representation of $C_{f}$ is a $k$-algebra homomorphism $\phi: C_{f} \rightarrow M_{m}(F)$, where $F$ is a field extension of $k$. The integer $m$ is called the dimension of the representation.

Let $C$ be the curve in $\mathbb{P}^{2}$ defined by the equation $w^{d}=f(u, v)$, where $u, v$ and $w$ are the projective coordinates. We assume that the binary form $f(u, v)$ does not have any repeated factors over an algebraic closure of $k$ and that the characteristic of $k$ does not divide $d$. With these assumptions, we have the following lemma:

Lemma 2.8. $C$ is a smooth curve of degree $d$.

Proof. It is obvious that $C$ is a curve of degree $d$. To prove that it is smooth, consider the partial derivatives of the defining equation $w^{d}-f(u, v)$ :

$$
\begin{aligned}
& \partial_{w}\left(w^{d}-f(u, v)\right)=d w^{d-1} \\
& \partial_{u}\left(w^{d}-f(u, v)\right)=-\partial_{u} f(u, v) \\
& \partial_{v}\left(w^{d}-f(u, v)\right)=-\partial_{v} f(u, v)
\end{aligned}
$$

It is now obvious that for a point $[u: v: w] \in C$ to be singular, $f(u, v)$ and both its partial derivatives have to vanish on it. But since we assumed that $f(u, v)$ has no repeated factors, this is not possible.

From now on, $C$ will denote this curve. We note that the genus of $C$ is $g=$ $(d-1)(d-2) / 2$. Assuming that $d \geq 4$, we have $g \geq 2$. We also note that the map $[u: v: w] \mapsto[u: v]$ defines a degree $d$ map $p: C \rightarrow \mathbb{P}^{1}$.

We now prove that the rank of a representation of $C_{f}$ is divisible by $d$ :

Proposition 2.9. (21, Proposition 1.1) Let $f$ be a binary form of degree $d$ over an infinite field $k$ with no repeated factors over an algebraic closure of $k$. If $\phi$ is a representation of the Clifford algebra $C_{f}$, then the degree $d$ of $f$ divides the rank of $\phi$.

We now want to describe representations of $C_{f}$ in more detail. Let $\phi: C_{f} \rightarrow$ $M_{m}(k)$ be a representation. Let $R=k[u, v]$ have the standard grading and let $S=k[u, v, w] /\left(w^{d}-f(u, v)\right)$. Note that $X=\operatorname{Proj} S$, and the map $p$ is induced by the inclusion $R \rightarrow S$. Let $\alpha_{u}=\phi(u)$ and $\alpha_{v}=\phi(v)$. These two matrices define a map of graded algebras $\phi_{f}: S \rightarrow M_{m}(R)$ by sending $u$ to $u I_{m}, v$ to $v I_{m}$ and $w$ to $u \alpha_{u}+v \alpha_{v}$. Conversely, if we have such a map, we can define a representation of $f$ by taking $\phi_{f}\left(u I_{m}\right)$ and $\phi_{f}\left(v I_{m}\right)$. Via this map, $R^{m}$ becomes a graded $S$-module. In this way, we get a vector bundle $E$ over $X$ such that $p_{*} E$ is trivial of rank $m$. This vector bundle $E$ also satisfies $H^{0}(E(-1))=0$. 
2.3. Azumaya algebras. We follow the discussions in [19] and 12 for this review. Let $A$ be an algebra over a commutative ring $R$. We assume that $R$ is the center of $A$. Then $A$ is called Azumaya over $R$ if $A$ is faithful, finitely generated and projective as an $R$-module and the map $\phi_{A}: A \otimes_{R} A^{\circ} \rightarrow \operatorname{End}_{R}(A)$ defined by

$$
\phi_{A}\left(\sum_{i} r_{i} \otimes s_{i}\right)(r)=\sum_{i} r_{i} r s_{i}
$$

is an isomorphism.

The following proposition will be useful later:

Proposition 2.10. Let $A$ and $B$ be Azumaya algebras over $R$. Then $A \otimes_{R} B$ is Azumaya over $R$.

By a construction similar to obtaining a quasicoherent sheaf over Spec $R$ using an $R$-module $M$, given an Azumaya algebra $A$ over $R$, we can obtain a sheaf of algebras over Spec $R$. We can use this as the motivation for the definition:

Definition 2.11. Let $X$ be a scheme. An $\mathcal{O}_{X}$-algebra $A$ is called an Azumaya algebra over $X$ if it is coherent as an $\mathcal{O}_{X}$-module and if, for all closed points $x$ of $X, A_{x}$ is an Azumaya algebra over the local $\operatorname{ring} \mathcal{O}_{X, x}$.

The conditions in Definition 2.11 imply that $A$ is locally free of finite rank as an $\mathcal{O}_{X}$-module.

Instead of using the definition to prove that an $\mathcal{O}_{X}$-algebra is Azumaya, we will make use of the following proposition:

Proposition 2.12. Let $A$ be an $\mathcal{O}_{X}$-algebra that is of finite type as an $\mathcal{O}_{X}$-module. Then $A$ is an Azumaya algebra over $X$ if and only if there is a flat covering $\left(U_{i} \rightarrow\right.$ $X)$ of $X$ such that for each $i, A \otimes_{\mathcal{O}_{X}} \mathcal{O}_{U_{i}} \cong M_{r_{i}}\left(\mathcal{O}_{U_{i}}\right)$ for some $r_{i}$.

Definition 2.13. If $S$ is a $k$-scheme then an $S$-representation of dimension $n$ of $B$ is a pair $\left(\phi, \mathcal{O}_{A}\right)$, where $\mathcal{O}_{A}$ is a sheaf of Azumaya algebras of rank $n^{2}$ over $S$ and $\phi: B \rightarrow H^{0}\left(S, \mathcal{O}_{A}\right)$ is a ring homomorphism. Two representations $\left(\phi_{1}, \mathcal{O}_{A_{1}}\right)$ and $\left(\phi_{2}, \mathcal{O}_{A_{2}}\right)$ are called equivalent if there is an isomorphism $\theta: \mathcal{O}_{A_{1}} \rightarrow \mathcal{O}_{A_{2}}$ of sheaves of rings such that $\phi_{2}=H^{0}(S, \theta) \circ \phi_{1}$. A representation of $B$ is called irreducible if the image of $B$ generates $\mathcal{O}_{A}$ locally. Let $\operatorname{Rep}_{n}(B, S)$ be the set of equivalence classes of irreducible $S$-representations of degree $n$ of $B$. This defines a contravariant functor $\mathcal{R} e p_{n}(B,-)$ from the category of $k$-schemes to the category of sets.

Theorem 2.14. (Theorem 4.1, 23]) The functor $\mathcal{R e p}_{n}(B,-)$ is representable in $(S c h / k)$.

Sometimes it is more convenient and easier to work with representation into endomorphism sheaves of vector bundles. Let $\mathcal{G}_{n}(B,-)$ be the subfunctor of $\mathcal{R} e p_{n}(B, S)$ consisting of representations of endomorphism sheaves of vector bundles of rank $n$. This is not a sheaf with respect to the flat topology. However, its sheafification with respect to the flat topology is well-known:

Lemma 2.15. (Lemma 4.2, [23) $\mathcal{R} e p_{n}(B,-) \cong \overline{\mathcal{G}}_{n}(B,-)$, where $\overline{\mathcal{G}}_{n}(B,-)$ denotes the sheafification of $\mathcal{G}_{n}(B,-)$.

We end this section with a lemma that will be useful later. Let $S$ be a scheme, and $\mathcal{O}$ be a sheaf of $\mathcal{O}_{S}$-algebras over $S$. A collection of global sections $\left(\sigma_{i} \in\right.$ $\left.H^{0}(S, \mathcal{O})\right)_{i \in I}$ is said to generate $\mathcal{O}$ if the stalks $\left(\sigma_{i}\right)_{s}$ generate $\mathcal{O}_{s}$ for all $s \in S$. 
Lemma 2.16. Let $S$ and $\mathcal{O}$ be as above. If the $\sigma_{i}(s)$ generate the fibers $\mathcal{O}(s)$ for closed points $s \in S$, then the $\sigma_{i}$ generate $\mathcal{O}$.

Proof. The statement is local in $S$, so we will assume that $S=\operatorname{Spec} R$ for a ring $R$ and that $\mathcal{O}=A^{\sim}$ for an $R$-algebra $A$.

First, we claim that if the $\sigma_{i}(s)$ generate $\mathcal{O}_{s}$ for closed points $s$ as a $k(s)$-algebra, then the $\left(\sigma_{i}\right)_{s}$ generate $\mathcal{O}_{s}$ as an $R_{s}$-algebra. But this follows from Nakayama's lemma.

Second, we claim that if the $\left(\sigma_{i}\right)_{s}$ generate $\mathcal{O}_{s}$ for closed $s$, then they generate $\mathcal{O}_{s}$ for all $s \in S$. The $\sigma_{i}$ correspond to elements $a_{i} \in A$. We know that for all maximal ideals $\mathfrak{m}$ in $R,\left(a_{i}\right)_{\mathfrak{m}}$ generate $A_{\mathfrak{m}}$ as an $R_{\mathfrak{m}}$-algebra.

Let $B$ be the $R$-subalgebra of $A$ generated by the $a_{i}$. Then $B_{\mathfrak{m}}=A_{\mathfrak{m}}$. By Corollary 2.9, [1], $A=B$ and the lemma is proved.

\section{Construction of the Universal Representation}

In this section, we construct a sheaf of Azumaya algebras over the variety $U$ defined in the previous section. Recall that there is a coarse moduli space $\mathcal{M}(r, D)$ of vector bundles over the curve $C$ with rank $r$ and degree $D$; this is a quasiprojective variety. The variety $U$ is the open subset of $\mathcal{M}(r, D)$ consisting of stable vector bundles $E$ over $C$ such that $H^{0}(E(-1))=0$.

We first recall how to construct quotients of vector bundles. Let $Y$ be an integral algebraic variety and $G$ an algebraic group acting on $Y$. We have the following definition:

Definition 3.1. (Definition 8.4.3, [10.) An algebraic vector $G$-bundle $F \rightarrow Y$ is a vector bundle over $Y$, equipped with a $G$-action which is linear in each fiber and such that the diagram

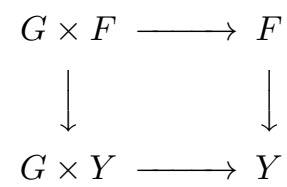

commutes. In other words, for every $y \in Y$, we have a linear map $F_{y} \rightarrow F_{g . y}$

$F$ is said to descend to $M$ if there is a vector bundle $F^{\prime}$ over $M$ such that the algebraic vector $G$-bundles $F$ and $\pi^{*} F^{\prime}$ are isomorphic.

This definition can also be stated in terms of sheaves. Let $\mathcal{F}$ denote the sheaf of sections of the vector bundle $F$. For every $g \in G$ and every open subset $V$ of $Y$, we have a linear map $\mathcal{F}(V) \rightarrow \mathcal{F}(g . V)$. These maps are required to satisfy the obvious compatibility conditions.

The following lemma gives a necessary and sufficient condition for an algebraic vector $G$-bundle $F$ to descend to $M$ : (For the proof, see Theorem 2.3, [14].)

Lemma 3.2. Let $G, Y$ and $M$ be as before. Let $F \rightarrow Y$ be an algebraic vector $G$-bundle over $Y$. Then $F$ descends to $M$ if and only if for every closed point $y$ of $Y$ such that the orbit of $y$ is closed, the stabilizer of $G$ at $y$ acts trivially on $F_{y}$.

Recall that $\Omega$ is the subset of the Quot-scheme $Q$ consisting of vector bundles $E$ over the curve $C$ such that $H^{0}(E(-1))=0$. We also have the bundle $\mathcal{E}$ parametrizing quotients of the trivial vector bundle $\mathbb{E}$ having the given Hilbert polynomial $P$. 
Recall also that $G L(N)$ acts on $\Omega$, and the stabilizers of points are the scalar matrices. Hence, there is an induced action of $P G L(N)$ on $\Omega$, and the good quotient is the variety $U$.

When we try to take the quotient of $\mathcal{E}$ by $P G L(N)$, however; we are unable to define an action of $P G L(N)$ on $\mathcal{E}$ because of the fact that the scalar multiples of identity in $G L(N)$ do not act trivially.

We resolve this difficulty as follows. Consider the direct image of $\mathcal{E}$ under the projection $\pi: C \times \Omega \rightarrow \Omega$. Then consider the endomorphism bundle $\mathcal{E} n d\left(\pi_{*} \mathcal{E}\right)$ and then define an action of $P G L(N)$ on it. (We prove that $\pi_{*} \mathcal{E}$ is a vector bundle below in Lemma 3.4.) Since $G L(N)$ only acts on the second component of $C \times \Omega$, this gives a $G L(N)$-action on $\pi_{*} \mathcal{E}$. Now let $G L(N)$ act on $\mathcal{E} n d\left(\pi_{*} \mathcal{E}\right)$ by conjugation, see below that the action of $G L(N)$ descends to a $P G L(N)$-action: The action of the stabilizer of a point on the fibers of $\pi_{*} \mathcal{E}$ is by scalar multiplication, but on $\mathcal{E} n d\left(\pi_{*} \mathcal{E}\right)$, this action becomes trivial. So the action of $G L(N)$ on $\mathcal{E} n d\left(\pi_{*} \mathcal{E}\right)$ descends to $P G L(N)$.

To be precise, let $g \in G L(N), f \in \Gamma\left(V, \mathcal{E} n d\left(\pi_{*} \mathcal{E}\right)\right)$ for an open subset $V \subset \Omega$. Then $f$ is an endomorphism of $\pi_{*} \mathcal{E}$ over $V$, and we define $g . f$ to be an endomorphism of $\pi_{*} \mathcal{E}$ over $g . V$ as follows. Let $s$ be a section of $\pi_{*} \mathcal{E}$ over an open subset $W \subset V$. Then $g . f$ is the endomorphism of $\pi_{*} \mathcal{E}$ that sends $s$ to $g\left(f\left(g^{-1} s\right)\right)$. It is obvious that this defines a $G L(N)$-action on $\mathcal{E} n d\left(\pi_{*} \mathcal{E}\right)$. Since a scalar matrix $\lambda I$ acts as multiplication by $\lambda$ on $\left(\pi_{*} \mathcal{E}\right)_{x}$, it can easily be seen as acting trivially on $\mathcal{E} n d\left(\pi_{*} \mathcal{E}\right)_{x}$ :

$$
\begin{aligned}
(\lambda I \cdot f)(s) & =\left(\lambda I \circ f \circ(\lambda I)^{-1}\right)(s) \\
& =\lambda I \circ f\left(\frac{1}{\lambda} s\right) \\
& =\lambda I \circ \frac{1}{\lambda} f(s) \\
& =\lambda \frac{1}{\lambda} f(s) \\
& =f(s) .
\end{aligned}
$$

This gives us a $P G L(N)$ action on $\mathcal{E} n d\left(\pi_{*} \mathcal{E}\right)$. As seen above, we have a quotient vector bundle $\mathcal{E} n d\left(\pi_{*} \mathcal{E}\right)^{P G L(N)}$, which we will denote as $\mathcal{A}$ over $U$.

Recall that there is a canonical map $q: C \rightarrow \mathbb{P}^{1}$, corresponding to the inclusion $k[u, v] \rightarrow k[u, v, w] /\left(w^{d}-f(u, v)\right)$. Recall that for a closed point $y$ in $\Omega \mathcal{E}_{y}$ denotes the pull-back of $\mathcal{E}$ under the canonical map $i d \times i_{y}: C \times \operatorname{Spec} k(y) \rightarrow C \times \Omega$.

We prove the following proposition, which follows from a standard result of Grothendieck.

Proposition 3.3. The coherent sheaf $q_{*}\left(\mathcal{E}_{y}\right)$ is isomorphic to the trivial bundle $\bigoplus_{i=1}^{r d} \mathcal{O}_{\mathbb{P}_{k(y)}^{1}}$.

Proof. We consider the case of an algebraically closed base field $k$ first. Since $\mathbb{P}_{k}^{1}$ is a nonsingular projective curve, any torsion-free coherent sheaf is a vector bundle. Since $q_{*}$ respects torsion-freeness, $q_{*}\left(\mathcal{E}_{y}\right)$ is a vector bundle on $\mathbb{P}_{k}^{1}$. It has rank $r d$ since $\mathcal{E}_{y}$ has rank $r$ and the map $q$ has degree $d$. Since any vector bundle on $\mathbb{P}_{k}^{1}$ is a sum of line bundles, we can write $q_{*}\left(\mathcal{E}_{y}\right) \cong \bigoplus_{i=1}^{r d} \mathcal{O}_{\mathbb{P}_{k}^{1}}\left(n_{i}\right)$ for some integers $n_{i}$. We 
have $\left.\chi\left(q_{*} \mathcal{E}_{y}\right)\right)=\chi\left(\mathcal{E}_{y}\right)$. Then,

$$
\begin{gathered}
\chi\left(q_{*}\left(\mathcal{E}_{y}\right)\right)=\left(\operatorname{rk}\left(q_{*}\left(\mathcal{E}_{y}\right)\right)\right)\left(1-g_{\mathbb{P}_{k}^{1}}\right)+\operatorname{deg}\left(q_{*}\left(\mathcal{E}_{y}\right)\right)=r d+\sum_{i=1}^{r d} n_{i}, \\
\chi\left(\mathcal{E}_{y}\right)=\left(\operatorname{rk}\left(\mathcal{E}_{y}\right)\right)(1-g)+\operatorname{deg}\left(\mathcal{E}_{y}\right)=r(1-g)+r(d+g-1)=r d .
\end{gathered}
$$

So we have $\sum_{i=1}^{r d} n_{i}=0$.

We also have, by the projection formula:

$$
h^{0}\left(\mathbb{P}^{1}, q_{*}\left(\mathcal{E}_{y} \otimes_{\mathcal{O}_{C}} \mathcal{O}_{C}(-1)\right)\right)=h^{0}\left(\mathbb{P}^{1},\left(q_{*} \mathcal{E}_{y}\right)(-1)\right)=h^{0}\left(C, \mathcal{E}_{y}(-1)\right)=0 .
$$

So it follows that $h^{0}\left(\mathbb{P}^{1},\left(q_{*} \mathcal{E}_{y}\right)(-1)\right)=0$. This is equal to $h^{0}\left(\mathbb{P}^{1}, \bigoplus_{i=1}^{r d} \mathcal{O}_{\mathbb{P}^{1}}\left(n_{i}-1\right)\right)$. It then follows that $n_{i}-1<0$ for all $i$ and hence $n_{i}=0$ because the sum of the $n_{i}$ is equal to 0 .

Now assume that $k$ is an arbitrary field, and let $\bar{k}$ denote its algebraic closure. Consider the pullback of $\mathcal{E}_{y}$ along the canonical morphism $i_{C}: C \times \bar{k} \rightarrow C \times k(y)$. Then we have $\left(q_{\bar{k}}\right)_{*}\left(i_{C}^{*}\left(\mathcal{E}_{y}\right)\right) \cong \bigoplus_{r d} \mathcal{O}_{\mathbb{P}^{1}}$. By Proposition 9.3, Chapter 3, [8]; we have $\left(q_{\bar{k}}\right)_{*}\left(i_{C}^{*}\left(\mathcal{E}_{y}\right)\right) \cong\left(i_{\mathbb{P}^{1}}^{*}\right)\left(q_{k(y)}\right)_{*} \mathcal{E}_{y}$. But we have $\operatorname{Aut}\left(\left(i_{\mathbb{P}^{1}}^{*}\right)\left(q_{k(y)}\right)_{*} \mathcal{E}_{y}\right)=G L_{r d}(\bar{k})$ and $H^{1}\left(G a l_{\bar{k} / k(y)}, G L_{r d}(\bar{k})\right)=0$ by Hilbert 90 . So we have $q_{*}\left(\mathcal{E}_{y}\right) \cong \bigoplus_{i=1}^{r d} \mathcal{O}_{\mathbb{P}_{k(y)}^{1}}$.

We can use this result to prove the following result, which is due to Kulkarni. (See Proposition 3.5, 9.

Lemma 3.4. Let $\pi: C \times \Omega \rightarrow \Omega$ denote the projection onto the second factor. Then $\pi_{*} \mathcal{E}$ is a locally free sheaf of rank $r d$.

Proof. For any point $y$ in $\Omega$, let $k(y)$ be the residue field of $y$. Denote by $\mathcal{E}_{y}$ the vector bundle $\left(i d \times i_{y}\right)^{*} \mathcal{E}$ on $C_{k(y)}$, where $i_{y}$ is the inclusion of the point $y$; that is, Spec $k(y) \rightarrow \Omega$. We have to prove that $\operatorname{dim}_{k(y)} H^{0}\left(C_{k(y)}, \mathcal{E}_{y}\right)$ is constant, and equal to $r d$. Recall that $\Omega$ is irreducible, and reduced. So, by Corollary 2 pg. 50, 13, it will follow that $\pi_{*} \mathcal{E}$ is a locally free sheaf of rank $r d$.

If $y$ is closed, then $q_{*} \mathcal{E}_{y} \cong \bigoplus_{i=1}^{r d} \mathcal{O}_{\mathbb{P}^{1}}$ by Proposition 3.3. So $h^{0}\left(C, \mathcal{E}_{y}\right)=$ $h^{0}\left(\mathbb{P}^{1}, q_{*} \mathcal{E}_{y}\right)=r d$. It is also well-known that the function $y \mapsto \operatorname{dim}_{k(y)} H^{0}\left(C_{k(y)}, \mathcal{E}_{y}\right)$ is upper semicontinuous. (See, for example, Theorem 12.8 in Chapter 3, [8].) Together with the fact that $\Omega$ is a Jacobson scheme (since it is locally of finite type over the spectrum of a field), it follows that $\operatorname{dim}_{k(y)} H^{0}\left(C_{k(y)}, \mathcal{E}_{y}\right)$ is constant and equal to $r d$ for all $y \in \Omega$. This implies that $\pi_{*} \mathcal{E}$ is a vector bundle of rank $r d$.

Lemma 3.5. Let $S$ be a scheme, and let $f: S \rightarrow \Omega$ be a morphism. Consider the commutative diagram:

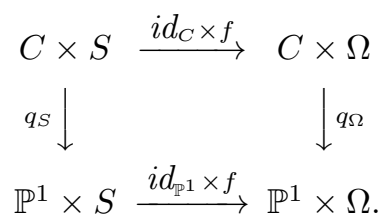

Then the coherent sheaves $\left(i d_{\mathbb{P}^{1}} \times f\right)^{*}\left(q_{\Omega}\right)_{*} \mathcal{E}$ and $\left(q_{S}\right)_{*}\left(i d_{C} \times f\right)^{*} \mathcal{E}$ are isomorphic.

Proof. Since $\mathcal{E}$ is a coherent sheaf on $C \times \Omega=\operatorname{Proj}\left(\mathcal{O}_{\Omega}[u, v, w] /\left(w^{d}-f\right)\right)$, there exists a sheaf of graded $\mathcal{O}_{\Omega}[u, v, w] /\left(w^{d}-f\right)$-modules $M$ such that $\mathcal{E}$ is isomorphic to $\widetilde{M}$. (See [5], II, Sections 3.2 and 3.3.) Then we have the following isomorphisms, as sheaves of $\mathcal{O}_{\Omega}[u, v]$-modules:

$$
\left(i d_{\mathbb{P}^{1}} \times f\right)^{*}\left(q_{\Omega}\right)_{*} \mathcal{E} \cong\left(\mathcal{O}_{S}[u, v] \otimes_{\mathcal{O}_{\Omega}[u, v]} M_{\mathcal{O}_{\Omega}[u, v]}\right)^{\sim},
$$


and

$\left(q_{k(y)}\right)_{*}\left(i d_{C} \times f\right)^{*} \mathcal{E} \cong\left(\mathcal{O}_{S}[u, v, w] /\left(w^{d}-f(u, v)\right) \otimes_{\mathcal{O}_{\Omega}[u, v, w] /\left(w^{d}-f(u, v)\right)} M\right)_{\mathcal{O}_{S}[u, v]}$.

But the two graded $k(y)[u, v]$-modules on the right side of the equations above are isomorphic. So the lemma is proved.

For the remainder of this section, we construct a map $\psi: C_{f} \rightarrow H^{0}(\mathcal{A})$ that is a universal representation for $C_{f}$. The next theorem is crucial in this construction. It is proven in 8] that for any morphism $g: X \rightarrow Y$ of schemes and a sheaf $\mathcal{G}$ of $\mathcal{O}_{X}$-modules, there is a natural morphism $g^{*} g_{*} \mathcal{G} \rightarrow \mathcal{G}$. This is a consequence of the adjointness of the functors $g^{*}$ and $g_{*}$. The proof of the following theorem follows the discussion in 9 closely. (See, Proposition 3.8, 9].)

Theorem 3.6. Let $\mathcal{F}=\left(q_{\Omega}\right)_{*} \mathcal{E}$. Then the natural morphism $u: p_{\Omega}^{*}\left(p_{\Omega}\right)_{*} \mathcal{F} \rightarrow \mathcal{F}$ is an isomorphism. (Recall that $p_{\Omega}$ is the canonical projection $\mathbb{P}^{1} \times \Omega \rightarrow \Omega$.)

Proof. We know that $\left(p_{\Omega}\right)_{*} \mathcal{F}=\pi_{*} \mathcal{E}$ is a locally free sheaf of rank $r d$ by Lemma 3.4. So the sheaf $p_{\Omega}^{*}\left(p_{\Omega}\right)_{*} \mathcal{F}$ is also a locally free sheaf of rank $r d$ on $\mathbb{P}_{\Omega}^{1}$.

First we claim that $\mathcal{F}$ is a locally free sheaf of rank $r d$ on $\mathbb{P}_{\Omega}^{1}$. We prove that $\operatorname{dim}_{k(y)} \mathcal{F} \otimes k(y)$ is $r d$ for any closed point $y$ in $\mathbb{P}_{\Omega}^{1}$. This will be sufficient by the upper semicontinuity of the dimension function and by the fact that $\mathbb{P}_{\Omega}^{1}$ is locally of finite type over $k$.

For any closed point $y$ in $\mathbb{P}^{1} \times \Omega$, consider the following commutative diagram:

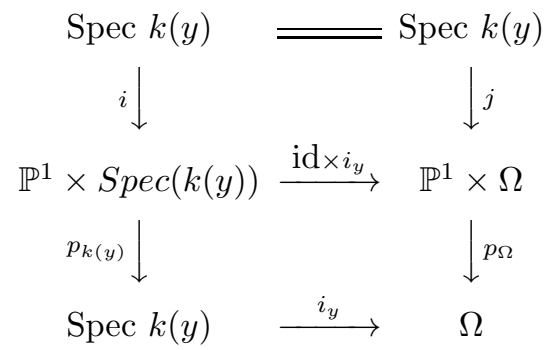

We prove that $\operatorname{dim}_{k(y)} \mathcal{F} \otimes_{\mathcal{O}_{\mathbb{P} 1 \times \Omega}} k(y)=\operatorname{dim}_{k(y)} j^{*} \mathcal{F}$ is $r d$. But $\operatorname{dim}_{k(y)} j^{*} \mathcal{F}=$ $\operatorname{dim}_{k(y)} i^{*}\left(i d \times i_{y}\right)^{*} \mathcal{F}$. From Lemma 3.5 and Proposition 3.3, it follows that $(i d \times$ $\left.i_{y}\right)^{*} \mathcal{F} \cong\left(i d \times i_{y}\right)^{*}\left(q_{\Omega}\right)_{*} \mathcal{E} \cong q_{*}(i d \times i)^{*} \mathcal{E} \cong q_{*}\left(\mathcal{E}_{y}\right)$ is a trivial vector bundle of rank $r d$, so that $\operatorname{dim}_{k(y)} i^{*}\left(i d \times i_{y}\right)^{*} \mathcal{F}$ is $r d$.

Now $u$ is a morphism of vector bundles of rank $r d$. To prove that it is an isomorphism, it is enough to show that $u_{x}:\left(p_{\Omega}^{*}\left(p_{\Omega}\right)_{*} \mathcal{F}\right)_{x} \rightarrow(\mathcal{F})_{x}$ is bijective for all points $x$ in $\mathbb{P}^{1} \times \Omega$. But it is sufficient to prove this only for closed points ([5], I, Corollary 0.5.5.7).

By ([5], I, Corollary 0.5.5.6) it is sufficient to prove that

$$
u_{y} \otimes i d:\left(p_{\Omega}^{*}\left(p_{\Omega}\right)_{*} \mathcal{F}\right)_{y} / \mathfrak{m}_{y}\left(p_{\Omega}^{*}\left(p_{\Omega}\right)_{*} \mathcal{F}\right)_{y} \rightarrow \mathcal{F}_{y} / \mathfrak{m}_{y} \mathcal{F}_{y}
$$

is surjective for all closed points $y$ in $\mathbb{P}_{\Omega}^{1}$. But this homomorphism is surjective if and only if the morphism

$$
j^{*} u: j^{*} p_{\Omega}^{*}\left(p_{\Omega}\right)_{*} \mathcal{F} \rightarrow j^{*} \mathcal{F}
$$

is surjective, which is the same as

$$
i^{*}\left(i d \times i_{y}\right)^{*} u: i^{*}\left(i d \times i_{y}\right)^{*} p_{\Omega}^{*}\left(p_{\Omega}\right)_{*} \mathcal{F} \rightarrow i^{*}\left(i d \times i_{y}\right)^{*} \mathcal{F}
$$


being surjective. So it is sufficient to prove that

$$
\left(i d \times i_{y}\right)^{*} u:\left(i d \times i_{y}\right)^{*} p_{\Omega}^{*}\left(p_{\Omega}\right)_{*} \mathcal{F} \rightarrow\left(i d \times i_{y}\right)^{*} \mathcal{F}
$$

is an isomorphism. But we have the isomorphism

$$
\left(i d \times i_{y}\right)^{*} p_{\Omega}^{*}\left(p_{\Omega}\right)_{*} \mathcal{F} \cong p_{k(y)}^{*} i_{y}^{*}\left(p_{\Omega}\right)_{*} \mathcal{F} .
$$

Note that $i_{y}^{*}\left(p_{\Omega}\right)_{*} \mathcal{F}$ is a trivial vector bundle of rank $r d$, and so

$$
p_{k(y)}^{*} i_{y}^{*}\left(p_{\Omega}\right)_{*} \mathcal{F} \cong p_{k(y)}^{*}\left(\bigoplus_{r d} \mathcal{O}_{S p e c k(y)}\right) \cong \bigoplus_{r d} \mathcal{O}_{\mathbb{P}_{k(y)}^{1}}
$$

This proves that $\left(i d \times i_{y}\right)^{*} p_{\Omega}^{*}\left(p_{\Omega}\right)_{*} \mathcal{F}$ is a trivial vector bundle. Recall that $\left(i d \times i_{y}\right)^{*} \mathcal{F}$ is a trivial vector bundle of rank $r d$. So the morphism $\left(i d \times i_{y}\right)^{*} u$ will be an isomorphism if it is so on the global sections. But the morphism

$$
p_{k(y)}^{*} i_{y}^{*}\left(p_{\Omega}\right)_{*} \mathcal{F} \rightarrow\left(i d \times i_{y}\right)^{*} \mathcal{F}
$$

on the global sections is an isomorphism if the natural morphism

$$
\left(p_{\Omega}\right)_{*} \mathcal{F} \otimes_{\mathcal{O}_{\Omega}} k(y) \rightarrow H^{0}\left(\mathbb{P}_{k(y)}^{1}, \mathcal{F}_{y}\right)
$$

is an isomorphism. By the earlier part, and Corollary 2, p. 50, [13, this is the case.

Recall that we have the morphisms $q_{\Omega}: C \times \Omega=C_{\Omega} \rightarrow \mathbb{P}^{1} \times \Omega=\mathbb{P}^{1}$ and $p_{\Omega}$ : $\mathbb{P}_{\Omega}^{1} \rightarrow \Omega$. Consider $\mathcal{O}_{\Omega}[u, v]$ and $\mathcal{O}_{\Omega}[u, v, w] /\left(w^{d}-f\right)$. These are sheaves of graded $\mathcal{O}_{\Omega}$-algebras generated by degree 1 elements. We can define their homogeneous spectra $\operatorname{Proj}\left(\mathcal{O}_{\Omega}[u, v]\right)=\mathbb{P}_{\Omega}^{1}$ and $\operatorname{Proj}\left(\mathcal{O}_{\Omega}[u, v, w] /\left(w^{d}-f\right)\right)=C \times \Omega$. Recall also that we have the vector bundle $\mathcal{F}$ on $\mathbb{P}_{\Omega}^{1}$. That this is a vector bundle follows from Lemma 3.4 and Theorem 3.6 .

Let $\mathcal{G}$ be a sheaf of $\mathcal{O}_{\mathbb{P}_{\Omega}^{1}}$-modules. Define

$$
\Gamma_{*}(\mathcal{G})=\bigoplus_{n \in \mathbb{Z}}\left(p_{\Omega}\right)_{*}(\mathcal{G}(n)) .
$$

In particular, we have

$$
\Gamma_{*}\left(\mathcal{O}_{\mathbb{P}_{\Omega}^{1}}\right)=\bigoplus_{n \in \mathbb{Z}}\left(p_{\Omega}\right)_{*}\left(\mathcal{O}_{\mathbb{P}_{\Omega}^{1}}(n)\right) .
$$

$\Gamma_{*}\left(\mathcal{O}_{\mathbb{P}_{\Omega}^{1}}\right)$ is then a sheaf of graded $\mathcal{O}_{\mathbb{P}_{\Omega}^{1}}$-algebras and $\Gamma_{*}(\mathcal{G})$ becomes a sheaf of graded $\Gamma_{*}\left(\mathcal{O}_{\mathbb{P}_{\Omega}^{1}}\right)$-modules.

Remark 3.7. These are particular cases of constructions carried out in [5], II, 3.3.

By Proposition 7.11, Chapter 2, 8], we have

$$
\Gamma_{*}\left(\mathcal{O}_{\mathbb{P}_{\Omega}^{1}}\right) \cong \mathcal{O}_{\Omega}[u, v]
$$

as sheaves of graded $\mathcal{O}_{\Omega}$-modules.

In the next proposition, we use Theorem 3.6 to describe $\Gamma_{*}(\mathcal{F})$.

Proposition 3.8. The $\mathcal{O}_{\Omega}[u, v]$-module $\Gamma_{*}(\mathcal{F})=\bigoplus_{n \in \mathbb{Z}}\left(p_{\Omega}\right)_{*}(\mathcal{F}(n))$ is isomorphic to $\left(\pi_{*} \mathcal{E}\right) \otimes_{\mathcal{O}_{\Omega}} \mathcal{O}_{\Omega}[u, v]$ as a sheaf of graded $\mathcal{O}_{\Omega}[u, v]$-modules. 
Proof. By the theorem, it is enough to compute the graded module associated to the coherent sheaf $p_{\Omega}^{*}\left(p_{\Omega}\right)_{*} \mathcal{F}$. By projection formula, we have

$$
\left(p_{\Omega}\right)_{*}(\mathcal{F}(n))=\left(p_{\Omega}\right)_{*}(\mathcal{F}) \otimes_{\mathcal{O}_{\Omega}}\left(p_{\Omega}\right)_{*}\left(\mathcal{O}_{\mathbb{P}_{\Omega}^{1}}(n)\right)
$$

By definition:

$$
\begin{aligned}
\Gamma_{*}(\mathcal{F}) & =\bigoplus_{n \in \mathbb{Z}}\left(p_{\Omega}\right)_{*}(\mathcal{F}(n)) \\
& =\bigoplus_{n \in \mathbb{Z}}\left(\left(p_{\Omega}\right)_{*}(\mathcal{F}) \otimes_{\mathcal{O}_{\Omega}}\left(p_{\Omega}\right)_{*}\left(\mathcal{O}_{\mathbb{P}_{\Omega}^{1}}(n)\right)\right) \\
& \cong\left(p_{\Omega}\right)_{*}(\mathcal{F}) \otimes_{\mathcal{O}_{\Omega}}\left(\bigoplus_{n \in \mathbb{Z}}\left(p_{\Omega}\right)_{*}\left(\mathcal{O}_{\mathbb{P}_{\Omega}^{1}}(n)\right)\right) \\
& =\left(p_{\Omega}\right)_{*}(\mathcal{F}) \otimes_{\mathcal{O}_{\Omega}} \Gamma_{*}\left(\mathcal{O}_{\mathbb{P}_{\Omega}^{1}}\right) \\
& \cong\left(\pi_{*} \mathcal{E}\right) \otimes_{\mathcal{O}_{\Omega}} \mathcal{O}_{\Omega}[u, v]
\end{aligned}
$$

as desired.

Next we consider the question of the existence of the universal representation. This representation will be an algebra homomorphism $\psi: C_{f} \rightarrow H^{0}(U, \mathcal{A})$ that satisfies a universal property that will be discussed in the next section. Recall that $\mathcal{A}$ is the Azumaya algebra obtained by taking the quotient of $\mathcal{E} n d\left(\pi_{*} \mathcal{E}\right)$ by the action of $P G L(N)$.

Theorem 3.9. There exists an algebra homomorphism $\psi: C_{f} \rightarrow H^{0}(U, \mathcal{A})$.

Proof. We prove this theorem by showing the existence of elements in $H^{0}(U, \mathcal{A})$ that satisfy the relations of the Clifford algebra.

First we make a simple observation. Let $\pi_{*} \mathcal{E}$ be as above, and let $\mathcal{M}$ be the graded $\mathcal{O}_{\Omega}[u, v]$-module $\pi_{*} \mathcal{E} \otimes_{\mathcal{O}_{\Omega}} \mathcal{O}_{\Omega}[u, v]$. Note that $\mathcal{M}_{0}=\pi_{*} \mathcal{E} . \pi_{*} \mathcal{E}$ is an $\mathcal{O}_{\Omega^{-}}$ module and the grading is determined by assigning $u$ and $v$ as degree 1 elements. Let $\mathcal{M}_{i}$ be the $i^{\text {th }}$ graded piece of $\mathcal{M}$. Then we have

$$
\operatorname{Hom}_{\mathcal{O}_{\Omega}}\left(\mathcal{M}_{0}, \mathcal{M}_{1}\right)=u E n d_{\mathcal{O}_{\Omega}}\left(\mathcal{M}_{0}\right)+v E n d_{\mathcal{O}_{\Omega}}\left(\mathcal{M}_{0}\right)
$$

This follows from the fact that $\mathcal{M}_{1}=u \mathcal{M}_{0} \oplus v \mathcal{M}_{0}$.

Consider the vector bundle $\mathcal{E}$ on $C_{\Omega}$. We have

$$
\begin{aligned}
\Gamma_{*}(\mathcal{E}) & =\bigoplus_{n \in \mathbb{Z}} \pi_{*}(\mathcal{E}(n)) \\
& =\bigoplus_{n \in \mathbb{Z}}\left(p_{\Omega}\right)_{*}\left(q_{\Omega}\right)_{*}(\mathcal{E}(n)) \\
& =\bigoplus_{n \in \mathbb{Z}}\left(p_{\Omega}\right)_{*}\left(\left(q_{\Omega}\right)_{*}(\mathcal{E}) \otimes_{\mathcal{O}_{\mathbb{P}_{\Omega}^{1}}} \mathcal{O}_{\mathbb{P}_{\Omega}^{1}}(n)\right) \\
& =\bigoplus_{n \in \mathbb{Z}}\left(p_{\Omega}\right)_{*}(\mathcal{F}(n)) \\
& =\Gamma_{*}(\mathcal{F})
\end{aligned}
$$

Hence, $\Gamma_{*}(\mathcal{F})$, which we proved to be isomorphic to $\left(\pi_{*} \mathcal{E}\right) \otimes_{\mathcal{O}_{\Omega}} \mathcal{O}_{\Omega}[u, v]$, can also be viewed as a sheaf of graded modules over $\Gamma_{*}\left(\mathcal{O}_{C_{\Omega}}\right) \cong \mathcal{O}_{\Omega}[u, v, w] /\left(w^{d}-f\right)$. Now $w$ is a homogeneous element of degree one in this graded $\mathcal{O}_{\Omega}$-algebra, so we can view $w$ (to be precise, the multiplication map by $w$ ) as an element of $H_{o m_{\mathcal{O}_{\Omega}}}\left(\mathcal{M}_{0}, \mathcal{M}_{1}\right)$. 
By the comment above, there exist elements $\alpha_{u}$ and $\alpha_{v}$ in $\operatorname{End}_{\mathcal{O}_{\Omega}}\left(M_{0}\right)$ such that we have the following equality in $\operatorname{Hom}_{\mathcal{O}_{\Omega}}\left(\mathcal{M}_{0}, \mathcal{M}_{1}\right)$ :

$$
w=u \alpha_{u}+v \alpha_{v} .
$$

Now we can consider the element $w^{d}$ as an element of $\operatorname{Hom}_{\mathcal{O}_{\Omega}}\left(M_{0}, M_{d}\right)$. The relation $w^{d}=f(u, v)$ holds in $H_{o m} m_{\Omega}\left(\mathcal{M}_{0}, \mathcal{M}_{d}\right)$ as well. This shows that the elements $\alpha_{u}$ and $\alpha_{v}$ in $\operatorname{End}_{\mathcal{O}_{\Omega}}\left(M_{0}\right)$ satisfy the relations of the Clifford algebra $C_{f}$. So we get a homomorphism:

$$
\chi: C_{f} \rightarrow \operatorname{End}_{\mathcal{O}_{\Omega}}\left(\mathcal{M}_{0}\right)
$$

Finally, recall that $P G L(N)$ acts on $E n d_{\mathcal{O}_{\Omega}}\left(\mathcal{M}_{0}\right)$, as was shown in the beginning of the section. Since $w$ is invariant under this action, it follows that $\alpha_{u}$ and $\alpha_{v}$ are also $P G L(N)$-invariant. Hence they give global sections of $\mathcal{A}$ that satisfy the relationships of the Clifford algebra; and that means that we have a map $\psi: C_{f} \rightarrow$ $H^{0}(\mathcal{A})$.

Now we prove the following proposition, which will be used in the proof of the main theorem.

Proposition 3.10. Suppose that $k$ is algebraically closed. Let $x \in U$ be a closed point, and $i_{x}:$ Spec $k \rightarrow U$ the inclusion. The pullback $i_{x}^{*}(\psi, \mathcal{A})$ is an $r d$ dimensional representation of $C_{f}$ that corresponds to the vector bundle $E$ over $C$ defined by the point $x$ under Van den Bergh's correspondence. (See Lemma 2, 22.)

Proof. Note that any closed point $x \in U$ can be lifted to a closed point $y \in \Omega$. We have the following diagram:

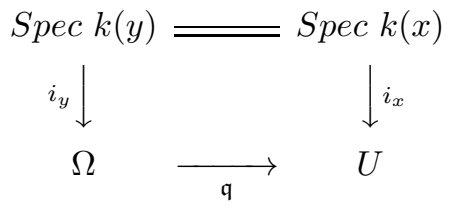

Recall that $\mathfrak{q}: \Omega \rightarrow U$ is the good quotient map by the action of $P G L(N)$. Hence, we have

$$
\begin{aligned}
i_{x}^{*} \mathcal{A} & =\left(\mathfrak{q} \circ i_{y}\right)^{*} \mathcal{A} \\
& \cong i_{y}^{*} \mathfrak{q}^{*} \mathcal{A} \\
& \cong i_{y}^{*} \mathcal{E} n d\left(\pi_{*} \mathcal{E}\right) \\
& \cong \mathcal{E} n d\left(i_{y}^{*} \pi_{*} \mathcal{E}\right)=\mathcal{E} n d\left(\left(\pi_{*} \mathcal{E}\right)_{y}\right)
\end{aligned}
$$

Since $\mathcal{E} n d\left(\pi_{*} \mathcal{E}\right)$ is an endomorphism bundle of dimension $(r d)^{2}, i_{x}^{*} \mathcal{A}$ also has dimension $(r d)^{2}$. Note also that $i_{x}^{*}\left(\alpha_{u}\right)$ and $i_{x}^{*}\left(\alpha_{v}\right)$ satisfy the relations of the Clifford algebra. This proves that $i_{x}^{*} \mathcal{A}$ is an $r d$-dimensional representation of $C_{f}$.

We claim that this is the same representation that corresponds to the point $x$ under Van den Bergh's correspondence. Recall the construction of a representation of $C_{f}$ from a stable vector bundle $E$ over $C$ with rank $r$, degree $r(d+g-1)$, and $H^{0}(E(-1))=0$ : Consider the direct image $q_{*}(E)$. This is a trivial vector bundle of rank $r d$ on $\mathbb{P}^{1}$ by Proposition 3.3. Its associated graded module over $k[u, v]$ is $\bigoplus_{r d} k[u, v]$, and this is also a graded module over $k[u, v, w] /\left(w^{d}-f(u, v)\right)$. The action of $w$ gives two matrices in $M_{r d}(k)$ satisfying the relations of the Clifford algebra and hence a map of algebras $C_{f} \rightarrow M_{r d}(k)$. Conversely, let $\phi$ be a representation of $C_{f}$. Consider the two matrices $\phi(u)$ and $\phi(v)$. The associated graded module of 
the trivial vector bundle of rank $r d$ on $\mathbb{P}^{1}$ over $k[u, v]$ is $\bigoplus_{r d} k[u, v]$. We can define an action of $w$ given by the images of the generators of the Clifford algebra, i.e. $w$ acts as $u \phi(u)+v \phi(v)$ on $\bigoplus_{r d} k[u, v]$. This makes $\bigoplus_{r d} k[u, v]$ into a graded module over $k[u, v, w] /\left(w^{d}-f\right)$. In this way, we get a stable rank $r$ vector bundle $E$ on the curve $C$, such that the degree of $E$ is $r(d+g-1)$ and $H^{0}(E(-1))=0$. (See Section 1, 22].)

Recall that the two sections $\alpha_{u}$ and $\alpha_{v}$ are defined by the action of $w$ on the $\mathcal{O}_{\Omega}[u, v, w] /\left(w^{d}-f\right)$-module $\Gamma_{*}(\mathcal{F})=\pi_{*} \mathcal{E} \otimes_{\mathcal{O}_{\Omega}} \mathcal{O}_{\Omega}[u, v]$. Therefore, the two sections $i_{y}^{*}\left(\alpha_{u}\right)$ and $i_{y}^{*}\left(\alpha_{v}\right)$ are determined by the action of $w$ on the $k(y)[u, v, w] /\left(w^{d}-f\right)$ module $\Gamma_{*}(\mathcal{F}) \otimes_{\mathcal{O}_{\Omega}} k(y)$.

Now we have

$$
\begin{aligned}
\Gamma_{*}(\mathcal{F}) \otimes_{\mathcal{O}_{\Omega}} k(y) & \cong\left(\pi_{*} \mathcal{E} \otimes_{\mathcal{O}_{\Omega}} \mathcal{O}_{\Omega}[u, v]\right) \otimes_{\mathcal{O}_{\Omega}} k(y) \\
& \cong\left(\pi_{*} \mathcal{E}\right)_{y} \otimes_{k(y)} k(y)[u, v]
\end{aligned}
$$

as graded $k(y)[u, v, w] /\left(w^{d}-f\right)$-modules and $\left(\pi_{*} \mathcal{E}\right)_{y}$, which is the restriction of $\pi_{*} \mathcal{E}$ to the closed point $y \in \Omega$, is a vector space of dimension $r d$. So we have $\Gamma_{*}(\mathcal{F}) \otimes_{\mathcal{O}_{\Omega}} k(y) \cong \bigoplus_{r d} k(y)[u, v]$.

Recall that any vector bundle corresponding to a closed point $y \in \Omega$ is such that its direct image under $q: C \rightarrow \mathbb{P}^{1}$ is trivial of rank $r d$. So the isomorphism class of $\mathcal{E}_{y}$ is determined by giving an action of $w$ on the $k(y)[u, v]$-module $\bigoplus_{r d} k(y)[u, v]$ such that $w^{d}=f(u, v)$. Since the two $w$-actions agree, the corresponding vector bundles are isomorphic.

We finish this section by proving that the $U$-representation $(\psi, \mathcal{A})$ is an irreducible $C_{f}$-representation as defined in Section 2.3 .

Proposition 3.11. The pair $(\psi, \mathcal{A})$ is an irreducible $U$-representation of dimension rd of the Clifford algebra $C_{f}$.

Proof. First we have to prove that $\mathcal{A}$ is an Azumaya algebra. Using Corollary 8.3.6 of [10, we can cover $U$ with étale maps $\rho_{i}: V_{i} \rightarrow U$ and $P G L(N)$-equivariant maps $\tau_{i}: V_{i} \times P G L(N) \rightarrow \Omega$ such that the diagrams

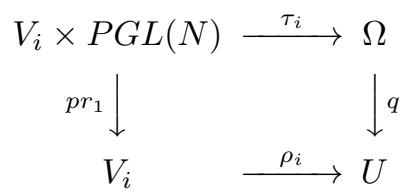

are cartesian.

Now it is obvious that the composition $\rho_{i} \circ p r_{1}$ is flat. The pullback of $\mathcal{A}$ along this map is isomorphic to the pullback of $\mathcal{A}$ along $q \circ \tau_{i}$. But we have:

$$
\begin{aligned}
\left(q \circ \tau_{i}\right)^{*} \mathcal{A} & \cong \tau_{i}^{*} q^{*} \mathcal{E} n d\left(\pi_{*} \mathcal{E}\right)^{P G L(N)} \\
& \cong \tau_{i}^{*} \mathcal{E} \operatorname{nd}\left(\pi_{*} \mathcal{E}\right) \\
& \cong \mathcal{E} n d\left(\tau_{i}^{*} \pi_{*} \mathcal{E}\right)
\end{aligned}
$$

It now follows from Proposition 2.12 that $\mathcal{A}$ is an Azumaya algebra. The fact that the dimension is $r d$ follows from the fact that the pullback of $\mathcal{A}$ along the quotient map $\Omega \rightarrow U$ is a rank $r d$ vector bundle. Finally, the map $\psi: C_{f} \rightarrow H^{0}(\mathcal{A})$ was constructed in Theorem 3.9 and irreducibility follows from Proposition 3.10 and Lemma 2.16 


\section{The Moduli Problem}

In this section, we assume $k$ to be algebraically closed.

As stated in the introduction, Procesi proved (see Theorem 1.8, Chapter 4, [17) that the functor $\mathcal{R} e p_{r d}\left(C_{f},-\right)$ is representable. In this section, we prove the main theorem; which states that $\mathcal{R e p}_{r d}\left(C_{f},-\right)$ is represented by $U$ and the $U$ representation $(\psi, \mathcal{A})$ as defined in the previous section. Recall that $U$ is defined to be the open subset of the moduli space $\mathcal{M}(r, r(d+g-1))$ consisting of stable vector bundles $E$ over $C$ such that $H^{0}(E(-1))=0$. These correspond to the irreducible representations of $C_{f}$. See [22. We constructed a sheaf of Azumaya algebras $\mathcal{A}$ on $U$ by considering the direct image $\pi_{*} \mathcal{E}$ of the vector bundle $\mathcal{E}$ on $C \times \Omega$ under the projection map $\pi: C \times \Omega \rightarrow \Omega$ and then considering the action of $P G L(N)$ on $\mathcal{E} n d\left(\pi_{*} \mathcal{E}\right)$. Taking the quotient gives us $\mathcal{A}$. In Theorem 3.9, we constructed an algebra homomorphism $\psi: C_{f} \rightarrow H^{0}(\mathcal{A})$ and in Proposition 3.11 we proved that this makes the pair $(\psi, \mathcal{A})$ into a $C_{f}$-representation. In this section, we will prove that this representation is the universal representation for the functor $\operatorname{Rep}_{r d}\left(C_{f},-\right)$.

If $S$ is a $k$-scheme, then by an $S$-representation of dimension $n$ of $C_{f}$ we mean a pair $\left(\psi, \mathcal{O}_{A}\right)$, where $\mathcal{O}_{A}$ is a sheaf of Azumaya algebras of dimension $n^{2}$ over $S$ and $\psi: C_{f} \rightarrow H^{0}\left(S, \mathcal{O}_{A}\right)$ is a $k$-algebra homomorphism. Two $S$-representations $\left(\psi_{1}, \mathcal{O}_{A_{1}}\right)$ and $\left(\psi_{2}, \mathcal{O}_{A_{2}}\right)$ are called equivalent if there is an isomorphism $\theta: \mathcal{O}_{A_{1}} \rightarrow$ $\mathcal{O}_{A_{2}}$ of sheaves of Azumaya algebras such that $\psi_{2}=H^{0}(S, \theta) \circ \psi_{1}$.

We call an $S$-representation of $C_{f}$ is called irreducible if the image of $C_{f}$ generates $\mathcal{O}_{A}$ locally.

Let $\mathcal{R} e p_{n}\left(C_{f},-\right)$ be the functor that assigns to a $k$-scheme $S$ the set of equivalence classes of irreducible $S$-representations of degree $n$ of $C_{f}$. Since Azumaya algebras pull back to Azumaya algebras and irreducible representations are stable under pull-back, it follows that $\operatorname{Rep}_{n}\left(C_{f},-\right)$ is indeed a functor.

It is known that this functor is representable in $S c h / k$. See, for example, Theorem 4.1, 23. Our goal in this section is to identify the scheme which represents it. Recall that we have the open subset $U$ of $\mathcal{M}(r, r(d+g-1))$ before and the sheaf of Azumaya algebras $\mathcal{A}=\mathcal{E} n d\left(\pi_{*} \mathcal{E}\right)^{P G L(N)}$ on it. We will prove that this pair represents the functor $\mathcal{R e p}_{r d}\left(C_{f},-\right)$; and here we use the morphism $\psi$ defined and proven to exist in the previous section. (See Theorem 3.9 and Proposition 3.11])

We also consider representations of $C_{f}$ into endomorphism sheaves of vector bundles. Let $\mathcal{G}_{r d}\left(C_{f},-\right)$ be the subfunctor of $\mathcal{R} e p_{r d}\left(C_{f},-\right)$ that assigns to a $k$-scheme $S$ the set of equivalence classes of irreducible $S$-representations into endomorphism sheaves of vector bundles of rank $r d$. Again, since endomorphism sheaves of vector bundles pull back to sheaves of the same kind, it follows that this is also a functor. It is not a sheaf (with respect to the fppf topology); however, it can be proven that its sheafification $\overline{\mathcal{G}_{r d}}$ is isomorphic to $\mathcal{R} e p_{r d}$. (See Lemma 4.2, [23]). This fact will be useful to us later when we prove the main theorem.

Let $\eta: C_{f} \rightarrow M_{r d}(K)$ be a representation of $C_{f}$, where $K$ is a field extension of $k$. Denote the images of the two generators of $C_{f}$ under $\eta$ by $\overline{\alpha_{u}}$ and $\overline{\alpha_{v}}$. Consider the morphism: 


$$
\begin{aligned}
S_{K}=\frac{K[u, v, w]}{\left(w^{d}-f(u, v)\right)} & \rightarrow M_{r d}(K[u, v]) \\
u, v & \mapsto u I_{r d}, v I_{r d} \\
w & \mapsto u \overline{\alpha_{u}}+v \overline{\alpha_{v}} .
\end{aligned}
$$

With the natural grading on $N=\bigoplus_{r d} K[u, v]$, the above morphism is a graded homomorphism. This makes $N$ into a graded $S_{K}$-module. So $\widetilde{N}$ is a (coherent) sheaf on $X=\operatorname{Proj} S_{K}=C_{K}$. Note that $\left(q_{K}\right)_{*} \widetilde{N} \cong \bigoplus_{r d} \mathcal{O}_{\mathbb{P}^{1}}$. (See the Introduction.) But we can prove more:

Lemma 4.1. (Lemma 4.3, 9.) $\tilde{N}$ is a rank $r$, degree $r(d+g-1)$ vector bundle on $X$. This bundle is stable, and it has $H^{0}(\widetilde{N}(-1))=0$.

Proof. For the first statement, by Proposition 2.5.1, Vol. 4 Part 2, and Lemma 12.3.1, Vol. 4 Part 3, [5]; it is enough to prove the statement for $\widetilde{N}$ with the assumption that $K$ is algebraically closed. We will prove that for any closed point $x \in X, \operatorname{dim}_{K}\left(\tilde{N} \otimes_{\mathcal{O}_{X, x}} K\right)=r$. By the usual upper semicontinuity argument, this is sufficient. Furthermore, it is obvious that $u$ and $v$ cannot be both in a homogeneous maximal ideal of $S_{K}$. So it is enough to prove the dimension condition above for any closed point $x$ in $X_{v}=\operatorname{Spec}\left(S_{K}\right)_{(v)}$, because the argument is the same for $X_{u}$. Now we have:

$$
\left(S_{K}\right)_{(v)}=\frac{K[\bar{u}, \bar{w}]}{\left(\bar{w}^{d}-f(\bar{u}, 1)\right)},
$$

and

$$
N_{(v)}=\bigoplus_{r d} K[\bar{u}] .
$$

Here, $\bar{u}=u / v$ and $\bar{w}=w / v . \bar{u}$ acts in a natural way and $\bar{w}$ acts as $\overline{u \alpha_{u}}+\overline{\alpha_{v}}$. Since $K$ is algebraically closed, any closed point in $\operatorname{Spec}\left(S_{K}\right)_{(v)}$ can be written as $\mathfrak{m}=(\bar{u}-a, \bar{w}-b)$ for some $a, b \in K$. So we have:

$$
\mathcal{O}_{\text {Spec }\left(S_{K}\right)_{(v)}, x} \cong\left(\frac{K[\bar{u}, \bar{w}]}{\left(\bar{w}^{d}-f(\bar{u}, 1)\right)}\right)_{(\bar{u}-a, \bar{w}-b)}
$$

and:

$$
\left(N_{(v)}\right)_{x} \cong \mathcal{O}_{x} \otimes_{\left(S_{K}\right)_{(v)}}\left(\bigoplus_{r d} K[\bar{u}]\right)
$$

Using these, we get:

$$
(\widetilde{N})_{x} \otimes_{\mathcal{O}_{X, x}} K \cong \frac{\bigoplus_{r d} K[\bar{u}]}{(\bar{u}-a, \bar{w}-b)\left(\bigoplus_{r d} K[\bar{u}]\right)} \cong \frac{\bigoplus_{r d} K}{\left(a \overline{\alpha_{u}}+\overline{\alpha_{v}}-b\right)\left(\bigoplus_{r d} K\right)}
$$

So the required dimension is $\operatorname{dim}_{K}\left(\operatorname{ker}\left(a \overline{\alpha_{u}}+\overline{\alpha_{v}}-b\right)\right)$. We have to prove that this dimension is equal to $r$. Let us assume that $f(a, 1) \neq 0$ at first. In this case, there are exactly $d$ points $(a, b)$ such that $b^{d}=f(a, 1)$. Over each of these points, the rank of the stalk $(\tilde{N})_{x} \otimes_{\mathcal{O}_{X, x}} K$ is at least $r$ by upper semicontinuity. Since they must add up to $r d$, each of them must be equal to $r$.

Consider $a \overline{\alpha_{u}}+\overline{\alpha_{v}} \in M_{r d}(K)$. We compute the dimension of its eigenspace of eigenvalue $b$. The characteristic polynomial of $a \overline{\alpha_{u}}+\overline{\alpha_{v}}$ is $t^{r d}-f(a, 1)$. This follows from the fact that when $f(a, 1) \neq 0$ all the roots are distinct (remember our initial assumption that $\operatorname{char}(K)$ does not divide $d$ ); and if $f(a, 1)=0$, then the matrix is nilpotent. Also, if $b \neq 0$, then $f(a, 1) \neq 0$ and $b$ is an eigenvalue of multiplicity 1 . 
Next, let $b=0$. Then we have $f(a, 1)=0$. We can find a matrix $B \in G L_{r d}(K)$ such that $B\left(a \overline{\alpha_{u}}+\overline{\alpha_{v}}\right) B^{-1}$ is in Jordan form. If $\operatorname{dim}_{K}\left(\operatorname{ker}\left(a \overline{\alpha_{u}}+\overline{\alpha_{v}}-b\right)\right)>1$, then we can write, for $w \in M_{r d}(K[u, v])$, det $w=\operatorname{det} B w B^{-1}=(a v-u)^{l} \operatorname{det} w^{\prime}$, where:

$$
B w B^{-1}=\left(\begin{array}{cccccc}
a v-u & \cdot & \cdot & \cdot & \cdot & \cdot \\
\cdot & \cdot & \cdot & \cdot & \cdot & \cdot \\
\cdot & \cdot & a v-u & \cdot & \cdot & \cdot \\
\cdot & \cdot & \cdot & 1 & \cdot & \cdot \\
\cdot & \cdot & \cdot & \cdot & \cdot & \cdot \\
\cdot & \cdot & \cdot & \cdot & \cdot & 1
\end{array}\right) w^{\prime} .
$$

There are $l$ diagonal entries $(a v-u)$ in the above matrix, and $l \geq r+1$. But then, we have $\operatorname{det} w^{d}=(a v-u)^{l d} \operatorname{det}\left(w^{\prime}\right)^{d}=f(u, v)^{d}$. But we immediately see that $(a v-u)$ is a repeated factor of $f(u, v)$ with multiplicity at least $l \geq 2$, which is a contradiction. So the required dimension condition is proved.

For the second part, consider the projection $q_{K}: C_{K} \rightarrow \mathbb{P}_{K}^{1}$. Then we have $\chi\left(C_{K}, \widetilde{N}\right)=\chi\left(\mathbb{P}_{K}^{1},\left(q_{K}\right)_{*} \widetilde{N}\right)$, and by Riemann-Roch:

$$
r(1-g)+\operatorname{deg}(\tilde{N})=r d(1-0)+\operatorname{deg}\left(\left(q_{K}\right)_{*} \tilde{N}\right) .
$$

$\operatorname{But}\left(q_{K}\right)_{*}(\tilde{N}) \cong \bigoplus_{r d} \mathcal{O}_{\mathbb{P}_{K}^{1}}$ and so its degree is 0 . This gives us deg $\widetilde{N}=r(d+g-1)$.

For the statement that $h^{0}\left(C_{K}, \widetilde{N}(-1)\right)=0$, note that we can use the projection formula to get:

$$
h^{0}\left(C_{K}, \widetilde{N}(-1)\right)=h^{0}\left(\mathbb{P}_{K}^{1},\left(q_{K}\right)_{*} \tilde{N}(-1)\right)=0 .
$$

Lastly, we have to prove that the vector bundle $\tilde{N}$ as constructed above is stable. For this, we follow the discussion in 22 . As in the introduction, we have a vector bundle $\widetilde{N}$ on $C$ such that $\left(q_{K}\right)_{*} \widetilde{N} \cong \mathcal{O}_{\mathbb{P}_{k}^{1}}^{r d}$. We will make use of the following formula:

$$
\begin{aligned}
\operatorname{deg}\left(\left(q_{K}\right)_{*} \widetilde{N}\right) / r k\left(\left(q_{K}\right)_{*} \widetilde{N}\right) & =\frac{\operatorname{deg}(\widetilde{N})-r k(\widetilde{N})(d+g-1)}{\operatorname{drk}(\widetilde{N})} \\
& =\frac{1}{d} \frac{\operatorname{deg}(\widetilde{N})}{r k(\widetilde{N})}+\frac{1-g}{d}-1
\end{aligned}
$$

Suppose $\widetilde{N}$ is strictly semistable. Then let $\mathcal{F} \subseteq \widetilde{N}$ be a subbundle such that $\operatorname{deg}(\mathcal{F}) / r k(\mathcal{F})=\operatorname{deg}(\widetilde{N}) / r k(\widetilde{N})$. It follows from a formula similar to 4.1 that $\operatorname{deg}\left(\left(q_{K}\right)_{*} \mathcal{F}\right)=0$. Recall that since $\left(q_{K}\right)_{*} \mathcal{F}$ is a subsheaf of the torsion-free sheaf $\left(q_{K}\right)_{*} \widetilde{N} \cong \mathcal{O}_{\mathbb{P}_{k}^{1}}^{r d}$, it is torsion-free itself and hence is a vector bundle. See Lemma 5.2.1, [10]. By Lemma 4.4.1, [10, it is a sum of line bundles on the projective line. Hence, $\left(q_{K}\right)_{*} \mathcal{F} \cong \bigoplus_{i=1}^{t} \mathcal{O}_{\mathbb{P}^{1}}\left(n_{i}\right)$, where $\sum n_{i}=0$. Now note that $H^{0}\left(\left(q_{K}\right)_{*} \widetilde{N}\right)=0$ and hence $H^{0}\left(\left(q_{K}\right)_{*} \mathcal{F}\right)=0$ as well. Hence, $n_{i} \leq 0$ for all $i$, and since $\sum n_{i}=0$, we have $n_{i}=0$ for all $i$. So we have $\left(q_{K}\right)_{*} \mathcal{F} \cong \mathcal{O}_{\mathbb{P}^{1}}^{t}$. It follows that the corresponding representation is reducible, which is contrary to our assumptions. This finishes the proof.

Next, we prove a relative version of Lemma 4.1. Let $S$ be a $k$-scheme, and let $(\psi, \mathcal{O})$ be an element of $\mathcal{G}_{r d}\left(C_{f}, S\right)$ so that $\mathcal{O}=\mathcal{E} n d_{\mathcal{O}_{S}}\left(\mathcal{E}^{S}\right)$ for some vector bundle 
$\mathcal{E}^{S}$ of rank $r d$ on $S$. We will first construct a rank $r$ vector bundle on $C \times_{k} S$. So consider the graded sheaf homomorphism

$$
\begin{aligned}
\frac{\mathcal{O}_{S}[u, v, w]}{w^{d}-f(u, v)} & \rightarrow \mathcal{E} n d\left(\mathcal{E}^{S}\right)[u, v] \\
u, v & \mapsto u, v \\
w & \mapsto u \psi(x)+v \psi(y)
\end{aligned}
$$

where $x$ and $y$ are the standard generators of $C_{f}$. This is a graded homomorphism of sheaves of $\mathcal{O}_{S}[u, v]$-algebras because the degree of $u$ and $v$ is 1 on the right side. We can view the right hand side of the above morphism as $\mathcal{E} d_{\mathcal{O}_{S}}\left(\mathcal{E}^{S} \otimes_{\mathcal{O}_{S}} \mathcal{O}_{S}[u, v]\right)$. So it allows us to view $\mathcal{E}^{S} \otimes_{\mathcal{O}_{S}} \mathcal{O}_{S}[u, v]$ as a sheaf of graded $\left(\mathcal{O}_{S}[u, v, w]\right) /\left(w^{d}-\right.$ $f(u, v)))$-modules. Since $C \times_{k} S \cong \operatorname{Proj}\left(\mathcal{O}_{S}[u, v, w]\right) /\left(w^{d}-f(u, v)\right)$, we get a sheaf $\mathcal{M}$ over $C \times_{k} S$. For the rest of the section, for any point $s$ in $S, q_{s}$ denotes the morphism $C \times_{k} k(s) \rightarrow \mathbb{P}_{k(s)}^{1}$ induced by the inclusion $k(s)[u, v] \rightarrow$ $(k(s)[u, v, w]) /\left(w^{d}-f(u, v)\right), p_{s}$ denotes the projection of the second factor of $\mathbb{P}_{k(s)}^{1}$ onto Spec $k(s)$, and $\pi_{s}$ denotes the composition $p_{s} \circ q_{s}$. We use a similar notation for an arbitrary field $K$ instead of $k(s)$.

Lemma 4.2. The sheaf $\mathcal{M}$ is a rank $r$ vector bundle on $C \times_{k} S$ of fiberwise constant degree of $r(d+g-1)$. Moreover, for any closed point $s \in S$, the rank $r$ vector bundle $\mathcal{M}_{s}=\mathcal{M} \otimes_{\mathcal{O}_{S}}$ Spec $k(s)$ on $C_{k(s)}$ is stable, has degree $r(d+g-1)$ and satisfies $h^{0}\left(C_{k(s)}, \mathcal{M}_{s}(-1)\right)=0$.

Proof. It is sufficient to prove these assertions when $S$ is affine. So let $S=$ Spec $R$. Let $C_{R}=C \times{ }_{k} S$. Assuming further (without loss of generality) that $\mathcal{E}$ is trivial on $S$, we have $H^{0}\left(S, \mathcal{E}^{S}\right) \cong \bigoplus_{r d} R$, because $\mathcal{E}$ is a vector bundle of rank $r d$. In this case, let us denote the graded $\left(R[u, v, w] /\left(w^{d}-f(u, v)\right)\right.$-module $H^{0}(S, \mathcal{E}) \otimes_{R} R[u, v]$ by $M$ and the corresponding sheaf $\mathcal{M}$ by $\widetilde{M}$.

Note that $\widetilde{M}$ is flat over $S$ and that $\pi: C \times S \rightarrow S$ is a flat morphism. So by Lemma 12.3.1, Vol. 4 Part 3, [5], it will be enough to prove that for any $s \in S, \widetilde{M}_{s}$ is a stable, rank $r$ vector bundle on $S$. Let $\widetilde{M}_{s}=\left(1 \times i_{s}\right)^{*} \widetilde{M}$, where $1 \times i_{s}$ is the morphism $C_{s}=C \times_{k} k(s) \rightarrow C \times_{k} S$. Consider the representation associated to the point $s$ via $\psi$ :

$$
\psi_{s}: C_{f} \rightarrow M_{r d}(k(s))
$$

Using Lemma 4.1, we obtain a sheaf $\widetilde{N}$ which is isomorphic to $\widetilde{M}_{s}$. So by Lemma 4.1 we know that $\widetilde{M}_{s}$ is a rank $r$, stable vector bundle. Also, along the fibers of the projection onto the second factor, the degree is $r(d+g-1)$.

The second assertion follows from Lemma 4.1 .

Let $S$ be a $k$-scheme and $\mathcal{V}$ be a vector bundle on $S$. Recall that $\mathcal{V}[u, v]^{\sim}$ defines a coherent sheaf on $\mathbb{P}_{S}^{1}$. It can be shown that $p_{S}^{*}(\mathcal{V})$ is isomorphic to $\mathcal{V}[u, v]^{\sim}$. (See, for example, Exercise III-47, 7].) We prove the following lemma which will be used in the main theorem.

Lemma 4.3. We have $\Gamma_{*}\left(\mathcal{V}[u, v]^{\sim}\right) \cong \mathcal{V}[u, v]$.

Proof. Using the definition of $\Gamma_{*}\left(\mathcal{V}[u, v]^{\sim}\right)$, we get

$$
\Gamma_{*}\left(\mathcal{V}[u, v]^{\sim}\right)=\bigoplus_{n \in \mathbb{Z}}\left(p_{S}\right)_{*}\left(\left(\mathcal{V}[u, v]^{\sim}\right)(n)\right)
$$




$$
\begin{aligned}
& \cong \bigoplus_{n \in \mathbb{Z}}\left(p_{S}\right)_{*}\left(p_{S}^{*}(\mathcal{V})(n)\right) \\
& \cong \bigoplus_{n \in \mathbb{Z}} \mathcal{V} \otimes\left(p_{S}\right)_{*}\left(\mathcal{O}_{\mathbb{P}_{S}^{1}}(n)\right) \\
& \cong \mathcal{V} \otimes \Gamma_{*}\left(\mathcal{O}_{\mathbb{P}_{S}^{1}}\right) \\
& \cong \mathcal{V} \otimes \mathcal{O}_{S}[u, v] \\
& \cong \mathcal{V}[u, v] .
\end{aligned}
$$

We are now in a position to prove the main theorem of this section:

Theorem 4.4. (Main Theorem) Any given degree $r d$ irreducible $S$-representation $\left(\psi, \mathcal{O}_{A}\right)$ can be obtained as the pull-back of the representation $\left(\psi, \mathcal{E} n d\left(\pi_{*} \mathcal{E}\right)^{P G L(N)}\right)$ by a unique map $f: S \rightarrow U$. In particular, $(\psi, \mathcal{A})$ represents the functor $\mathcal{R} e p_{r d}\left(C_{f},-\right)$.

Proof. It is already known (see Theorem 4.1, 23]) that the functor $\mathcal{R} e p_{r d}\left(C_{f},-\right)$ is representable. Let $T$ denote the scheme that represents it and let $(\Psi, \mathcal{B})$ be the universal representation. Since we have a $U$-representation $(\psi, \mathcal{A})$, we obtain a unique map $\alpha: U \rightarrow T$ such that $(\psi, \mathcal{A}) \cong \alpha^{*}(\Psi, \mathcal{B})$. We will construct another map $\beta: T \rightarrow U$ and prove that $\alpha$ and $\beta$ are inverses of each other.

Let $\left(\phi, \mathcal{E} n d\left(\mathcal{E}^{S}\right)\right)$ be a degree $r d S$-representation in $\mathcal{G}_{r d}\left(C_{f},-\right)$. Using Lemma 4.2. we can construct a vector bundle $\mathcal{M}$ on $C \times S$ such that for any point $s \in S$, $\mathcal{M}_{s}$ is stable, has rank $r$ and degree $r(d+g-1)$ and $H^{0}\left(\mathcal{M}_{s}(-1)\right)=0$. Recall that $\mathcal{M}(r, r(d+g-1))$ is a coarse moduli space of vector bundles and $U \subseteq \mathcal{M}(r, r(d+g-$ $1)$ ). So using the coarse moduli property, we obtain a map $f: S \rightarrow \mathcal{M}(r, r(d+g-1))$ whose image lies in $U$.

Since it will be necessary to use the vector bundle $\mathcal{E}$ on $C \times \Omega$ later in this proof, we use the local lifts of $f$ to $\Omega$. Using the local universal property of $\Omega$, we see that $S$ can be covered by Zariski open sets $S_{i}$ such that the restriction $f_{i}$ of $f$ to $S_{i}$ can be lifted (not uniquely) to $\Omega$. In other words, we get maps $g_{i}: S_{i} \rightarrow \Omega$ with $f_{i}=\mathfrak{q} \circ g_{i}$. (Recall that $\mathfrak{q}: \Omega \rightarrow U$ is the good quotient map.) These maps satisfy $\left(i d \times g_{i}\right)^{*} \mathcal{E} \cong \mathcal{M}_{i}$ on $S_{i}$, where $\mathcal{M}_{i}$ is the restriction of $\mathcal{M}$ to $C \times S_{i}$ :

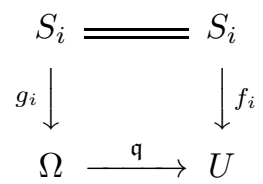

We claim that $g_{i}^{*}\left(\chi, \mathcal{E} n d\left(\pi_{*} \mathcal{E}\right)\right)$ is equivalent to (see Definition 2.13) $\left(\phi, \mathcal{E} n d\left(\mathcal{E}^{S_{i}}\right)\right)$, where $\mathcal{E}^{S_{i}}$ is the restriction of $\mathcal{E}^{S}$ to $C \times S_{i}$. To prove this claim, we will make extensive use of the following diagram of maps:

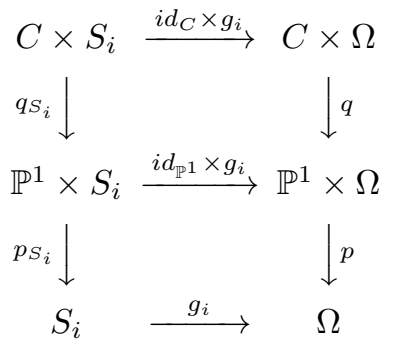


On top of this diagram, we have the vector bundle $\mathcal{M}_{i}$ constructed over $C \times S_{i}$ as in Lemma 4.2 and the bundle $\mathcal{E}$ on $C \times \Omega$ as in Grothendieck's theorem 2.6. In Lemma 4.2, $\mathcal{M}_{i}$ was defined to be $\left(\mathcal{E}^{S_{i}} \otimes_{\mathcal{O}_{S_{i}}} \mathcal{O}_{S_{i}}[u, v]\right)^{\sim}$, which was viewed as a graded $\left(\mathcal{O}_{S_{i}}[u, v, w]\right) /\left(w^{d}-f(u, v)\right)$-module. We can see from this that $\left(q_{S_{i}}\right)_{*} \mathcal{M}_{i} \cong$ $\mathcal{E}^{S_{i}}[u, v]^{\sim}$, viewed as a graded $\mathcal{O}_{S_{i}}[u, v]$-module. Next we compute $\Gamma_{*}\left(\mathcal{M}_{i}\right)$. Recall that this is a graded $\left(\mathcal{O}_{S_{i}}[u, v, w]\right) /\left(w^{d}-f(u, v)\right)$-module. Then we have

$$
\begin{aligned}
\Gamma_{*}\left(\mathcal{M}_{i}\right) & =\bigoplus_{n \in \mathbb{Z}}\left(p_{S_{i}}\right)_{*}\left(q_{S_{i}}\right)_{*}\left(\mathcal{M}_{i}(n)\right) \\
& \cong \bigoplus_{n \in \mathbb{Z}}\left(p_{S_{i}}\right)_{*}\left(\left(q_{S_{i}}\right)_{*}\left(\mathcal{M}_{i}\right) \otimes_{\mathcal{O}_{\mathbb{P}_{S_{i}}^{1}}} \mathcal{O}_{\mathbb{P}_{S_{i}}^{1}}(n)\right) \\
& \cong \Gamma_{*}\left(\left(q_{S_{i}}\right)_{*} \mathcal{M}_{i}\right) \\
& \cong \Gamma_{*}\left(\mathcal{E}^{S_{i}}[u, v]^{\sim}\right) \\
& \cong \mathcal{E}^{S_{i}}[u, v] .
\end{aligned}
$$

Here, the last line follows from Lemma 4.3. Similarly, we compute $\Gamma_{*}\left(\left(i d_{C} \times g_{i}\right)^{*} \mathcal{E}\right)$. Recall also that this is a graded $\left(\mathcal{O}_{S_{i}}[u, v, w]\right) /\left(w^{d}-f(u, v)\right)$-module.

$$
\begin{aligned}
\Gamma_{*}\left(\left(i d_{C} \times g_{i}\right)^{*} \mathcal{E}\right) & =\bigoplus_{n \in \mathbb{Z}} \pi_{*}\left(i d_{C} \times g_{i}\right)^{*} \mathcal{E}(n) \\
& \cong \bigoplus_{n \in \mathbb{Z}}\left(p_{S_{i}}\right)_{*}\left(q_{S_{i}}\right)_{*}\left(\left(\left(i d_{C} \times g_{i}\right)^{*} \mathcal{E}\right) \otimes_{\mathcal{O}_{C \times S_{i}}} \mathcal{O}_{C \times S_{i}}(n)\right) \\
& \cong \bigoplus_{n \in \mathbb{Z}}\left(p_{S_{i}}\right)_{*}\left(\left(q_{S_{i}}\right)_{*}\left(i d_{C} \times g_{i}\right)^{*} \mathcal{E} \otimes \mathcal{O}_{\mathbb{P}_{S_{i}}^{1}} \mathcal{O}_{\mathbb{P}_{S_{i}}^{1}}(n)\right) \\
& \cong \bigoplus_{n \in \mathbb{Z}}\left(p_{S_{i}}\right)_{*}\left(\left(i d_{\mathbb{P}_{S_{i}}^{1}} \times g_{i}\right)^{*}\left(q_{\Omega}\right)_{*} \mathcal{E} \otimes \mathcal{O}_{\mathbb{P}_{S_{i}}^{1}} \mathcal{O}_{\mathbb{P}_{S_{i}}^{1}}(n)\right) \\
& \cong \bigoplus_{n \in \mathbb{Z}}\left(p_{S_{i}}\right)_{*}\left(\left(i d_{\mathbb{P}_{S_{i}}^{1}} \times g_{i}\right)^{*}\left(p_{\Omega}\right)^{*}\left(\pi_{*} \mathcal{E}\right) \otimes_{\mathcal{O}_{\mathbb{P}_{S_{i}}^{1}}} \mathcal{O}_{\mathbb{P}_{S_{i}}^{1}}(n)\right) \\
& \cong \bigoplus_{n \in \mathbb{Z}}\left(p_{S_{i}}\right)_{*}\left(\left(p_{S_{i}}\right)^{*} g_{i}^{*}\left(\pi_{*} \mathcal{E}\right) \otimes \mathcal{O}_{\mathbb{P}_{S_{i}}^{1}} \mathcal{O}_{\mathbb{P}_{S_{i}}^{1}}(n)\right) \\
& \cong g_{i}^{*}\left(\pi_{*} \mathcal{E}\right) \otimes \bigoplus_{n \in \mathbb{Z}}\left(p_{S_{i}}\right)_{*}\left(\mathcal{O}_{\mathbb{P}_{S_{i}}^{1}}(n)\right) \\
& \cong g_{i}^{*}\left(\pi_{*} \mathcal{E}\right) \otimes \Gamma_{*}\left(\mathcal{O}_{\mathbb{P}_{S_{i}}^{1}}\right) \\
& \cong g_{i}^{*}\left(\pi_{*} \mathcal{E}\right) \otimes \mathcal{O}_{S_{i}}[u, v] \\
& \cong g_{i}^{*}\left(\pi_{*} \mathcal{E}\right)[u, v] .
\end{aligned}
$$

Since $\mathcal{M}_{i}$ and $\left(i d_{C} \times g_{i}\right)^{*} \mathcal{E}$ are isomorphic as sheaves, it follows that $\Gamma_{*}\left(\mathcal{M}_{i}\right)$ and $\Gamma_{*}\left(\left(i d_{C} \times g_{i}\right)^{*} \mathcal{E}\right)$ are isomorphic as $\left(\mathcal{O}_{S_{i}}[u, v, w]\right) /\left(w^{d}-f(u, v)\right)$-modules.

Recall that the representation $\left(\phi, \mathcal{E} n d\left(\mathcal{E}^{S_{i}}\right)\right)$ is defined by the action of $w$ on $\mathcal{E}^{S_{i}}[u, v]$ and the representation $g_{i}^{*}\left(\chi, \mathcal{E} n d\left(\pi_{*} \mathcal{E}\right)\right)$ is similarly defined by the action of $w$ on $g_{i}^{*}\left(\pi_{*} \mathcal{E}\right)[u, v]$. Now let $\eta: \Gamma_{*}\left(\mathcal{M}_{i}\right) \cong \mathcal{E}^{S_{i}}[u, v] \rightarrow \Gamma_{*}\left(\left(i d_{C} \times g_{i}\right)^{*} \mathcal{E}\right) \cong$ $g_{i}^{*} \pi_{*} \mathcal{E}[u, v]$ be an isomorphism of $\left(\mathcal{O}_{S_{i}}[u, v, w]\right) /\left(w^{d}-f(u, v)\right)$-modules. Let $w$ act on $\mathcal{E}^{S_{i}}$ by $u \phi_{u}^{1}+v \phi_{v}^{1}$ and on $g_{i}^{*} \pi_{*} \mathcal{E}$ by $u \phi_{u}^{2}+v \phi_{v}^{2}$. So we have

$$
\eta(w)=w \eta
$$

that is,

$$
\eta\left(\left(u \phi_{u}^{1}+v \phi_{v}^{1}\right)\right)=\left(u \phi_{u}^{2}+v \phi_{v}^{2}\right)(\eta)
$$


Comparing the $u$ and $v$ components, we see that $\eta \phi_{u}^{1} \eta^{-1}=\phi_{u}^{2}$ and $\eta \phi_{v}^{1} \eta^{-1}=\phi_{v}^{2}$. Hence the two representations $\left(\phi, \mathcal{E} n d\left(\mathcal{E}^{S_{i}}\right)\right)$ and $g_{i}^{*}\left(\chi, \mathcal{E} n d\left(\pi_{*} \mathcal{E}\right)\right)$ are equivalent.

Now we have

$$
\begin{aligned}
\left(\alpha \circ f_{i}\right)^{*}(\Psi, \mathcal{B}) & \cong f_{i}^{*}(\psi, \mathcal{A}) \\
& \cong g_{i}^{*} \mathfrak{q}^{*}(\psi, \mathcal{A}) \\
& \cong g_{i}^{*}\left(\chi, \mathcal{E} n d\left(\pi_{*} \mathcal{E}\right)\right) \\
& \cong\left(\phi, \mathcal{E} n d\left(\mathcal{E}^{S_{i}}\right)\right) .
\end{aligned}
$$

Next, define morphisms of functors as follows:

$$
\mathcal{G}_{r d}\left(C_{f}, S\right) \rightarrow \operatorname{Hom}_{S c h / k}(S, U) \rightarrow \operatorname{Hom}_{S c h / k}(S, T)
$$

by sending $\left(\phi, \mathcal{E} n d\left(\mathcal{E}^{S}\right)\right)$ to the map $f$ defined as above first, and then by sending $f$ to the composition $\alpha \circ f$. Hence the second map is induced by $\alpha: U \rightarrow T$. Recall that we are using the flat topology on $(S c h / S)$. Since the sheafification of $\mathcal{G}_{r d}\left(C_{f},-\right)$ is $\mathcal{R e p}_{r d}\left(C_{f},-\right)$, and $\operatorname{Hom}_{S c h / k}(-, T)$ is a sheaf; we get morphisms of sheaves as follows:

$$
\mathcal{R e p}_{r d}\left(C_{f},-\right) \cong \operatorname{Hom}_{S c h / k}(-, T) \rightarrow \operatorname{Hom}_{S c h / k}(-, U) \rightarrow \operatorname{Hom}_{S c h / k}(-, T) .
$$

This sequence induces a sequence of morphisms of schemes $T \rightarrow U \rightarrow T$; and any $\left(\phi, \mathcal{E} n d\left(\mathcal{E}^{S}\right)\right)$ is mapped to $\alpha \circ f$ by this composition. Recall that the second map is $\alpha$, and we will call the first map $\beta$.

Next, let $S$ be a $k$-scheme and let $(\gamma, \mathcal{G})$ be an $r d$-dimensional irreducible $S$ representation of $C_{f}$. Since $\mathcal{G}$ is Azumaya, by Proposition 2.12 we can cover $S$ by flat maps $S_{i} \rightarrow S$ such that the pullback of $(\gamma, \mathcal{G})$ is of the form $\left(\phi, \mathcal{E} n d\left(\mathcal{E}^{S_{i}}\right)\right)$ for some vector bundle $\mathcal{E}^{S_{i}}$ on $S_{i}$. This gives us a map $f_{i}: S_{i} \rightarrow U$ as stated earlier in the proof. Then we can cover $S_{i}$ by Zariski open subsets $S_{i, j}$ over which we can lift the restrictions $f_{i, j}: S_{i, j} \rightarrow U$ to $\Omega$. Following the formula 4.3, we see that the pullback of the universal representation $(\Psi, \mathcal{B})$ along $\alpha \circ f_{i, j}$ is isomorphic to $\left(\phi_{i, j}, \mathcal{E} n d\left(\mathcal{E}^{S_{i, j}}\right)\right)$. This means that given any section of the sheaf $\mathcal{R} e p_{r d}\left(C_{f},-\right)$ corresponding to an irreducible $r d$-dimensional representation $(\gamma, \mathcal{G})$ over a $k$-scheme $S$, there is a flat cover of $S$ by maps $S_{i, j} \rightarrow S$ such that the morphism of functors defined in the formula 4.7 maps the restriction of $(\gamma, \mathcal{G})$ to $S_{i, j}$ to the unique map $S_{i, j} \rightarrow T$ that pulls the universal representation $(\Psi, \mathcal{B})$ back to $(\gamma, \mathcal{G})$. Since $\operatorname{Hom}_{S c k / k}(-, T)$ is a sheaf, this proves that the composition in 4.7 is the identity. In other words, $\alpha \circ \beta$ is the identity.

Since $\alpha \circ \beta$ is the identity on $T$, and since $(\psi, \mathcal{A})$ pulls back to $(\Psi, \mathcal{B})$ under $\beta$, it is clear that $(\psi, \mathcal{A})$ must pull back to $(\Psi, \mathcal{B})$ under $\beta$.

We claim that $\beta$ is the inverse of $\alpha$. We only need to prove that the composition $\beta \circ \alpha: U \rightarrow T \rightarrow U$ is the identity on $U$. It is clear that the $U$-representation $(\psi, \mathcal{A})$ pulls back to itself under $\beta \circ \alpha$.

Denote $\delta=\beta \circ \alpha: U \rightarrow U$ for brevity. We claim that $\delta$ maps a closed point $x \in U$ to itself. To see this, consider the composition $\delta \circ i_{x}: \operatorname{Spec} k(x) \rightarrow U \rightarrow U$. Then $i_{x}^{*}(\psi, \mathcal{A})$ is the irreducible $r d$-dimensional representation of $C_{f}$ corresponding to the closed point $x$ by Proposition 3.10. Let $y=\delta(x)$, it is clear that the inclusion morphism of $y$ is $\delta \circ i_{x}$. We have

$$
\begin{aligned}
\left(\delta \circ i_{x}\right)^{*}(\psi, \mathcal{A}) & \cong i_{x}^{*} \delta^{*}(\psi, \mathcal{A}) \\
& \cong i_{x}^{*}(\psi, \mathcal{A})
\end{aligned}
$$


Again, by Proposition 3.10, y must be the closed point corresponding to the $r d$ dimensional representation $i_{x}^{*}(\psi, \mathcal{A})$. But this is the point $x$. It is now clear that the map $\delta$ is the identity on closed points. Since $U$ is a variety, it now follows from Lemma 1.2 that $\beta \circ \alpha$ is the identity map on $U$. This finishes the proof.

\section{Galois Descent}

In the last section, we proved Theorem 4.4 under the assumption that the base field $k$ is algebraically closed. In this section, we assume that the binary form $f(u, v)$ is defined over a perfect, infinite field $k$ whose characteristic does not divide $d$; and we prove the main theorem in this case.

We denote the algebraic closure of $k$ by $k^{\prime}$. Then $k^{\prime} / k$ is Galois. Let $G=$ $\operatorname{Gal}\left(k^{\prime} / k\right)$ denote the Galois group. Throughout this section, we denote the constructs with base field $k^{\prime}$ with a superscript ${ }^{\prime}$.

Let $V^{\prime}$ be a variety over $k^{\prime}$. Recall that a model for $V^{\prime}$ is a variety $V$ over $k$ such that $V^{\prime} \cong V \times_{k} k^{\prime}$.

Proposition 5.1. There is a model $U$ for $U^{\prime}$, where $U^{\prime}$ is the moduli space as constructed in Section 3 over $k^{\prime}$.

Proof. Recall (Théorème 3.1, 4]) that the Quot scheme $Q$ parametrizing quotients of $\mathbb{E}$ as defined in 2.1 is defined for an arbitrary base. We have an open subscheme $\Omega^{\prime}$ in $Q^{\prime}$. Since the canonical map $Q^{\prime} \rightarrow Q$ is open, the image $\Omega$ of $\Omega^{\prime}$ is open in $Q$. It is now clear that there is a $P G L(N)$-action on $\Omega$ that induces the $P G L(N)$-action on $\Omega^{\prime}$ and hence a uniform geometric quotient $U$ of $\Omega$ by $P G L(N)$ by Proposition 1.9, Chapter 1, 3. Since this quotient is uniform, we have $U^{\prime}=U \times k^{\prime}$.

We now describe how to obtain a $U$-representation $(\psi, \mathcal{A})$ that is a model for the universal representation. We consider the trivial bundle $\mathbb{E}$ over $k$ with rank $N$ as described in 2.1 and we consider the Quot scheme $Q$ over $k$ parametrizing the quotients of $\mathbb{E}$ that have rank $r$ and degree $r(d+g-1)$. (Recall that prescribing the rank and degree is equivalent to prescribing the Hilbert polynomial of a vector bundle by the Riemann-Roch theorem.) Consider the open subset $\Omega$ of $Q$ as described in Proposition 5.1 There is a universal quotient bundle $\mathcal{E}$ over $\Omega$ as described in Section 2.1. To show that $\left(\psi^{\prime}, \mathcal{A}^{\prime}\right)$ descends to $k$, we mimic the construction in Section 3. The proofs of Lemma 3.4 and Theorem 3.6 carry over. We can then follow the construction of the universal representation as in Theorem 3.9 to obtain a sheaf of Azumaya algebras $\mathcal{A}$ and a $k$-algebra homomorphism $\psi: C_{f} \rightarrow H^{0}(\mathcal{A})$.

We now show that $(\psi, \mathcal{A}) \times k^{\prime}$ is isomorphic to $\left(\psi^{\prime}, \mathcal{A}^{\prime}\right)$. By Proposition 9.3, Chapter 3, 8, we have $\pi_{*}^{\prime}\left(\mathcal{E}^{\prime}\right) \cong \pi_{*}(\mathcal{E}) \times k^{\prime}$, where $\pi^{\prime}: C \times \Omega^{\prime} \rightarrow \Omega^{\prime}$ or $\pi: C \times \Omega \rightarrow \Omega$ denotes the projection. It is now clear that $\mathcal{E} n d\left(\pi_{*}^{\prime} \mathcal{E}^{\prime}\right) \cong \mathcal{E} n d\left(\pi_{*} \mathcal{E}\right) \times k^{\prime}$. Since $\mathcal{A}^{\prime}$ is defined to be $\mathcal{E} n d\left(\pi_{*}^{\prime} \mathcal{E}^{\prime}\right)^{P G L(N)}$ and $\mathcal{A}$ is defined to be $\mathcal{E} n d\left(\pi_{*} \mathcal{E}\right)^{P G L(N)} ; \mathcal{A}^{\prime}$ descends to $\mathcal{A}$.

It now remains to show that the two sections $\alpha_{u}^{\prime}$ and $\alpha_{v}^{\prime}$ of $H^{0}\left(\mathcal{A}^{\prime}\right)$ defined in Theorem 3.9 descend to two sections $\alpha_{u}$ and $\alpha_{v}$ of $H^{0}(\mathcal{A})$. To see this, recall that $\alpha_{u}^{\prime}$ and $\alpha_{v}^{\prime}$ were defined by the action of $w$ (considered as an element of $\left.\mathcal{O}_{\Omega^{\prime}}[u, v, w] /\left(w^{d}-f\right)\right)$ on $\Gamma_{*}\left(\mathcal{F}^{\prime}\right)$. Here, $\mathcal{F}^{\prime}$ denotes the pushforward $\left(q_{\Omega^{\prime}}\right)_{*}\left(\mathcal{E}^{\prime}\right)$. Now, since the pushforward commutes with flat base change, we have $\left(q_{\Omega^{\prime}}\right)_{*}\left(\mathcal{E}^{\prime}\right) \cong$ $\left(q_{\Omega}\right)_{*}(\mathcal{E}) \times k^{\prime}$. This implies that $\Gamma_{*}\left(\mathcal{F}^{\prime}\right) \cong \Gamma_{*}(\mathcal{F})^{\prime}$. Consider the two sections $\alpha_{u}$ and $\alpha_{v}$ of $H^{0}(\mathcal{A})$ obtained by the action of $w$ on $\Gamma_{*}(\mathcal{F})$. Since $\Gamma_{*}\left(\mathcal{F}^{\prime}\right) \cong \Gamma_{*}(\mathcal{F})^{\prime}$,

and since the action of $w$ is compatible with base change, it follows that $\alpha_{u}^{\prime}$ and 
$\alpha_{v}^{\prime}$ descend to $\alpha_{u}$ and $\alpha_{v}$ respectively. This proves that $(\psi, \mathcal{A}) \times k^{\prime}$ is isomorphic to $\left(\psi^{\prime}, \mathcal{A}^{\prime}\right)$.

We now prove the main result of this section.

Theorem 5.2. $U$ represents $\mathcal{R} e p_{r d}$.

Proof. Let $S$ be a $k$-scheme. Denote $S^{\prime}=S \times_{k} k^{\prime}$. We have a map $\mathcal{R} e p_{r d}(S) \rightarrow$ $\mathcal{R} e p_{r d}^{\prime}\left(S^{\prime}\right)$ by taking an $S$-representation $\left(\psi, \mathcal{O}_{A}\right)$ to the $S^{\prime}$-representation $\left(\psi^{\prime}, \mathcal{O}_{A} \times_{k}\right.$ $\left.k^{\prime}\right)$ where $\psi^{\prime}$ is the induced $k^{\prime}$ algebra homomorphism $C_{f} \rightarrow H^{0}\left(\mathcal{O}_{A} \times_{k} k^{\prime}\right)$. It is clear that $\left(\psi^{\prime}, \mathcal{O}_{A} \times_{k} k^{\prime}\right)$ is invariant under the action of $G$. Hence we obtain a $\operatorname{map} \mathcal{R e p}_{r d}(S) \rightarrow \mathcal{R} e p_{r d}^{\prime}\left(S^{\prime}\right)^{G} \cong \operatorname{Hom}_{S c h / k^{\prime}}\left(S^{\prime}, U^{\prime}\right)^{G}=\operatorname{Hom}_{S c h / k}(S, U)$. Here, $G$ acts on $H_{o m} \operatorname{sch}_{k k^{\prime}}\left(S^{\prime}, U^{\prime}\right)$ by conjugation. In order to show that this map is a bijection, we need to show that the first arrow is a bijection. Injectivity follows from Theorem 6, pg. 135, [18. To prove surjectivity, consider an element $\left(\psi^{\prime}, \mathcal{O}_{A}^{\prime}\right)$ in $\mathcal{R} e p_{r d}^{\prime}\left(S^{\prime}\right)^{G}$. This means that, for every $\sigma \in G$ considered as an automorphism of $S^{\prime}, \sigma^{*}\left(\psi^{\prime}, \mathcal{O}_{A}^{\prime}\right)$ is equivalent to $\left(\psi^{\prime}, \mathcal{O}_{A}^{\prime}\right)$. In other words, we can find an isomorphism $\phi_{\sigma}: \sigma^{*} \mathcal{O}_{A}^{\prime} \rightarrow \mathcal{O}_{A}^{\prime}$ such that the map $\psi^{\prime}: C_{f} \rightarrow H^{0}\left(\mathcal{O}_{A}^{\prime}\right)$ is equal to the composition $H^{0}\left(\phi_{\sigma}\right) \circ \sigma^{*} \psi^{\prime}$. Here, $\sigma^{*} \psi^{\prime}$ is the induced map $C_{f} \rightarrow H^{0}\left(\sigma^{*} \mathcal{O}_{A}^{\prime}\right)$. Now consider $\phi_{\tau} \circ \tau \phi_{\sigma} \circ \phi_{\tau \sigma}^{-1}$. This is an automorphism of $\mathcal{O}_{A}^{\prime}$ that is the identity on the two global sections given by the images of $x_{1}$ and $x_{2}$ in $C_{f}$. Since these two global sections generate $\mathcal{O}_{A}^{\prime}$ locally, $\phi_{\tau} \circ \tau \phi_{\sigma} \circ \phi_{\tau \sigma}^{-1}$ must be the identity. Hence the $\phi_{\sigma}$ form a descent datum for $\left(\psi^{\prime}, \mathcal{O}_{A}^{\prime}\right)$ and there is an $S$-representation $\left(\psi, \mathcal{O}_{A}\right)$ that is a model for $\left(\psi^{\prime}, \mathcal{O}_{A}^{\prime}\right)$. This proves the surjectivity of the map as above and finishes the proof.

\section{REFERENCES}

[1] David Eisenbud, Commutative algebra with a view toward algebraic geometry, SpringerVerlag, 1999.

[2] Gerd Faltings, Vector bundles on curves (lecture notes), 1995.

[3] David Mumford; John Fogarty, Geometric invariant theory, 2nd edition, Springer-Verlag, 1982.

[4] Alexandre Grothendieck, Techniques de construction et théorèmes d'existence en géométrie algébrique iv: Les schémas de Hilbert, Séminaire Bourbaki (1961), no. 221.

[5] Alexandre Grothendieck, Éléments de géométrie algébrique, Institut des Hautes Etudes Scientifiques, 1965.

[6] D. Haile, On the clifford algebra of a binary cubic form, Amer. J. Math. 106, 1269-1280.

[7] D. Eisenbud; J. Harris, The geometry of schemes, Springer-Verlag, 2000.

[8] Robin Hartshorne, Algebraic geometry, Springer-Verlag, 1977.

[9] R. S. Kulkarni, On the clifford algebra of a binary form, Trans. Amer. Math. Soc. 355 (2003), 3181-3208.

[10] J. LePotier, Lectures on vector bundles, Cambridge University Press, 1997.

[11] H. B. Lawson; M.-L. Michelsohn, Spin geometry, Princeton University Press, 1989.

[12] J. S. Milne, Etale cohomology, Princeton University Press, 1980.

[13] David Mumford, Abelian varieties, 2nd ed., Oxford University Press, 1970.

[14] J.-M. Drezet; M. S. Narasimhan, Groupe de picard des varietes de modules de fibres semistables sur les courbes algebriques, Invent. Math. 97, 53-94.

[15] P. E. Newstead, Introduction to moduli problems and orbit spaces, Springer-Verlag, 1978.

[16] I. Porteous, Clifford algebras and the classical groups, Cambridge University Press, 1995.

[17] Claudio Procesi, Rings with polynomial identities, Marcel Dekker, 1973.

[18] S. Bosch; W. Lütkebohmert; M. Raynaud, Neron models, Springer-Verlag, 1990.

[19] David J. Saltman, Lectures on division algebras, American Mathematical Society, 1999.

[20] C. S. Seshadri, Space of unitary vector bundles on a compact riemann surface, Ann. of Math. 85 (1967), 303-336. 
[21] D. Haile; S. Tesser, On azumaya algebras arising from clifford algebras, J. Algebra 116 (1988), 372-384.

[22] M. van den Bergh, Linearisations of binary and ternary forms, J. Algebra 109 (1987), 172183.

[23] M. van den Bergh, The center of the generic division algebra, J. Algebra 127 (1989), 106-126.

120 Middlesex College, The University of Western Ontario, London, ON N6A 5B7 CANADA

E-mail address: ecoskun@uwo.ca 